\title{
Instabilités de liquéfaction et phénomène de mobilité cyclique dans les sables ${ }^{1}$
}

J. CANOU

N. BENAHMED

J.-C. DUPLA

V. DE GENNARO

CERMES

(centre commun

ENPC-LCPC)

ENPC, Cité Descartes

Champs-sur-Marne 77455 Marne-la-Vallée Cedex 2
On présente dans cet article les comportements mécaniques élémentaires qui sont à l'origine des ruptures par écoulement de massifs de sol sableux, souvent spectaculaires et de grande amplitude, connues sous le nom générique de liquéfaction. Après un bref rappel sur le comportement mécanique des sols sous chargement monotone, on décrit le phénomène dit de liquéfaction "statique », instabilité particullère caractéristique des sables lấches (contractants) sous sollicitation monotone non drainée (ou à volume constant). On passe ensuite au cas des sollicitations cycliques en mettant I'accent sur les analogies existant entre comportement monotone et cyclique et en distinguant deux phénomènes distincts, à savoir la liquéfaction "vraie», très similaire à la liquéfaction statique, caractéristique des sables uniquement contractants et la mobilité cyclique, quí se manifeste dans les sables dilatants. Ces deux phénomènes sont contrôlés par des mécanismes différents, et, pour un même sable, l'un ou l'autre des phénomènes peut être initié et se développer sous sollicitation cyclique, en fonction des conditions initiales caractérisant le matériau. On donne finalement quelques éléments relatifs à la recherche d'un cadre conceptuel global qui permettrait de rendre compte des différents comportements non drainés observables sous chargement monotone et cyclique.

Mots-clés : sable, cisaillement, triaxial, liquéfaction, mobilité cyclique, instabilité, rupture, état critique, état stable, écoulement, contractance, dilatance, pression interstitielle.

\section{Liquefaction instabilities and cyclic mobility phenomena in sands}

We present in this paper the elementary mechanical behaviours which are responsible for sometimes very spectacular flow failures of saturated sand masses, known under the generic term of liquefaction. After a brief description of the mechanical behaviour of solls under monotonic loading, we describe the phenomenon known as \& static p liquefaction, which is a specific instability characteristic of loose (contractant) sands submitted to monotonic undrained (or constant volume) loading. Then, the behaviour of sand under cyclic loading is examined, showing strong analogies with the monotonic case. Two distinct phenomena, which may be initiated under cyclic loading, are described, called cyclic mobility, characteristic of dilatant states and true liquefaction, characteristic of essentially contracting states. These phenomena are controlled by different mechanisms and, for a given sand, either one may be triggered and develop, in function of the initial conditions of the sand. Finally, elements are given on a possible general framework, which could allow to account, in the same state diagram, for the different undrained behaviours which may be observed under monotonic and cyclic loadings,

Key words : sand, shear, triaxial, liquefaction, cyclic mobility, instability, failure, critical state, steady state, flow, contractancy, dilatancy, pore water pressure. 


\section{Introduction}

La littérature géotechnique décrit de très nombreux exemples de sinistres associés à des ruptures de sol particulières et souvent spectaculaires, dans lesquelles celui-ci semble perdre soudainement une grande partie de sa résistance au cisaillement et s'écoule de manière semblable à un fluide épais, d’où le terme de liquéfaction, apparu très tôt dans la littérature (Hazen, 1920; Terzaghi, 1925: Terzaghi, 1956). Ce type de comportement est généralement observé dans le cas de matériaux sableux saturés (mais aussi dans certains types d'argiles), soumis à des sollicitations rapides en regard de la perméabilité du sol, monotones ou cycliques, telles que séismes, raz de marée, vidanges rapides, chocs, explosions, etc., et pour des configurations de sol propices à l'écoulement (déclivités). Dans certains cas, des écoulements quasi spontanés, parfois de très grande envergure, peuvent être initiés sans cause extérieure apparente et se développer selon un mécanisme régressif, alors que la configuration est telle (par exemple faible déclivité) que la stabilité (c classique ) du massif sableux (au sens de Mohr-Coulomb) est largement assurée. Lorsque de telles ruptures se produisent, les bâtiments ou les structures de génie civil fondées sur le sol en écoulement vont être entraînés avec celui-ci (enfoncement et basculement de bâtiments, déplacements latéraux d'ouvrages, rupture de barrages, etc.).

Pour ce qui est des ruptures "quasi spontanées " dans des massifs naturels, n'ayant pas d'origine sismique claire, on peut citer de nombreux glissements s'étant produits dans la province de Zeeland, aux PaysBas (Koppejan et al., 1948) de même que le long des berges du Mississipi aux Etats-Unis ou dans de nombreux fjords norvégiens (Bjerrum et al., 1961). En France, on peut citer le cas de la rupture du nouveau port de Nice en 1979 (Schlosser, 1985) et du port de Dunkerque (Blondeau, 1986). Pour ce qui est des massifs de sol artificiels, on peut mentionner des problèmes fréquents de stabilité liés à la mise en place de matériaux sableux par remblaiement hydraulique dans le cadre, par exemple du stockage de résidus miniers (Highter et Tobin, 1980) ou de la création d'îles artificielles ou de massifs sous-marins destinés à recevoir des infrastructures pétrolières (Sladen et al., 1985).

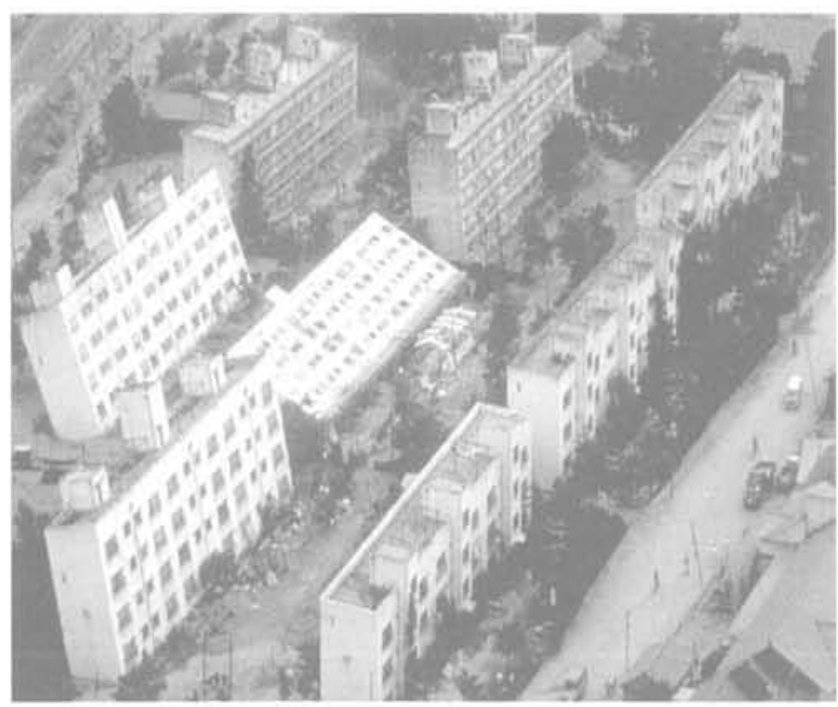

En ce qui concerne les ruptures ayant une origine sismique claire, de très nombreux cas ont été rapportés dans la littérature avec, en particulier, en 1964, le séisme de Niigata au Japon (Seed et Idriss, 1967) et celui d'Alaska (Seed, 1968). Ces deux séismes ont été à l'origine du développement de recherches importantes, en particulier aux États-Unis et au Japon, dans le domaine de la liquéfaction des sables. On peut aussi citer le séisme de San Fernando (Californie), en 1971, au cours duquel le barrage en terre Lower San Fernando s'est rompu par liquéfaction d'une partie même du corps de barrage formée de matériau sableux mis en place par remblaiement hydraulique. Plus récemment, les séismes de Loma Prieta (San Francisco, 1989), de Kobe (Japon, 1995) et d'Tzmit (Turquie, 1999) ont été, eux aussi, le siège de nombreux dégâts attribués à la liquéfaction des sables (Fig. 1).

Plusieurs définitions du phénomène de liquéfaction ont été chronologiquement proposées par différents auteurs, faisant parfois en fait référence à des phénomènes différents. II existe maintenant un consensus acceptable sur l'identité du phénomène, qui peut être résumé par la définition proposée par Sladen et al. (1985), qui rejoint celles données par Seed (1979) et par Castro and Poulos (1977) : « La liquéfaction est un phénomène dans lequel une masse de sol perd un pourcentage important de sa résistance au cisaillement, sous l'action d'un chargement monotone ou cyclique, quasi statique ou dynamique, et s'écoule de manière semblable à un liquide jusqu'à ce que les contraintes de cisaillement auxquelles est soumis le matériau puissent être équilibrées par sa résistance au cisaillement réduite.

Après un bref rappel sur les notions de base qui seront utilisées dans la suite du texte ainsi que sur les traits de comportement essentiels des sables en cisaillement monotone, on décrit ensuite le phénomène de liquéfaction des sables lâches sous chargement monotone (liquéfaction " statique $)$ ). On présente ensuite les éléments de base relatifs au comportement des sables sous cisaillement cyclique qui sont ensuite utilisés pour décrire les phénomènes de mobilité cyclique et de liquéfaction cyclique « vraie $)$. On présente finalement l'ébauche d'un cadre conceptuel global permettant de rendre compte, dans le plan des contraintes triaxial, des différents comportements non drainés observés.

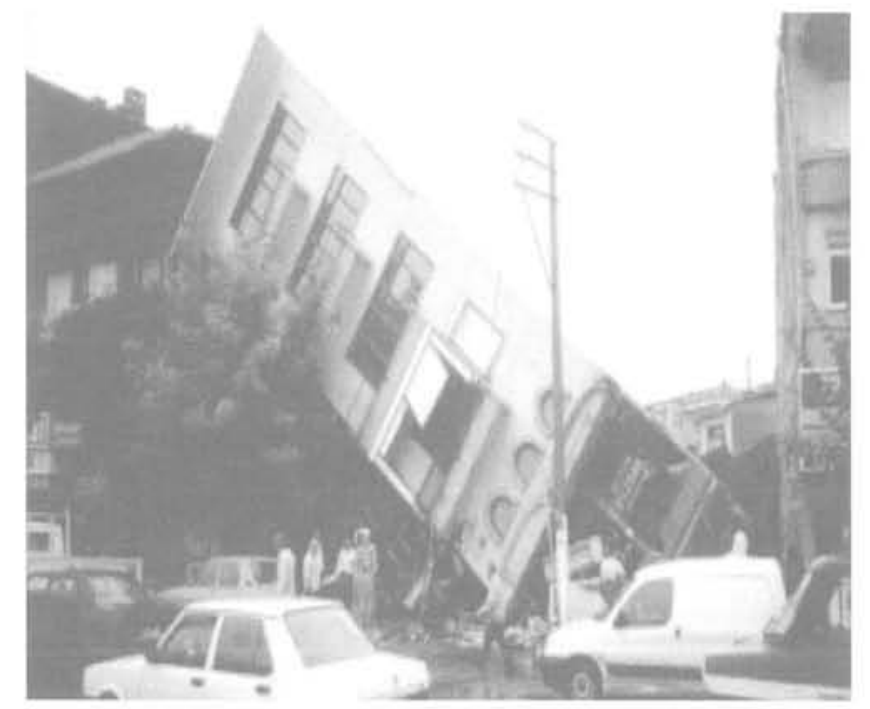

FG.1 Exemples de sinistres résultant de phénomènes de liquéfaction des sables (Niigata, 1964; Izmit, 1999). Examples of disasters induced by liquefaction of sand (Niigata, 1964; Izmit, 1999). 


\section{Généralités sur le comportement des sables en cisaillement monotone}

\section{1}

\section{Rappels}

On s'intéresse au cas des sables parfaitement saturés en eau, qui vont se déformer sous l'action des sollicitations appliquées. Le sol biphasique est assimilé à un milieu continu pour lequel on définit le tenseur des contraintes classique de la mécanique, $\underline{\sigma}$, que l'on décompose de manière usuelle, selon le postulat de Terzaghi, en la somme d'un tenseur des contraintes effectives, $\underline{\sigma}$, rendant compte de la part du chargement effectivement reprise par le squelette granulaire, et d'un tenseur isotrope, u.I (I étant la matrice identité), rendant compte de la part du chargement reprise par l'eau sous forme de pression interstitielle $u$ :

$$
\underline{\sigma}=\underline{\underline{\sigma}}+u .1
$$

Le comportement drainé parfait correspond à la réponse du matériau observable lorsque l'eau interstitielle peut s'écouler librement entre les grains au cours du chargement, sans développer de surpressions interstitielles. Il est donc caractérisé par une surpression interstitielle nulle $(\Delta \mathrm{u}=0)$ au cours de la sollicitation et des variations de volume $\Delta \mathrm{V}$ généralement non nulles du matériau.

Le comportement non drainé parfait correspond, au contraire, au cas où l'eau interstitielle reste « bloquée » au sein du matériau lors de l'application de la sollicitation, sans possibilité d'écoulement relatif par rapport aux grains. Ce sera le cas, sur site, de vitesses de sollicitations élevées, typiques de celles rencontrées dans les problèmes de liquéfaction (séismes, raz-de-marée, chocs, etc.). Des surpressions interstitielles vont alors se développer au sein du sol, entrainant une évolution des contraintes effectives qui pourra être très différente de celle des contraintes totales et le sol va alors se déformer à volume constant (aux compressibilités près des grains et de l'eau, qui sont généralement négligées devant celle du squelette).

Le comportement non drainé parfait est donc caractérisé par une non-variation de volume du sol et lé développement de surpressions interstitielles non nulles. Il correspond, en fait, au comportement du matériau à volume constant pour lequel le « lien » cinématique de non variation de volume est assuré par l'eau interstitielle. La même réponse pourra ètre observée sur un sable sec, pourvu que le dispositif d'essai utilisé soit alors capable d'assurer, par asservissement, une non-variation de volume du matériau (cas du triaxial vrai, Lanier et al., 1991 ; Darve, 1993).

Les propriétés de contractance et de dilatance, qui caractérisent la nature des déformations volumiques d'origine irréversible des sols sollicités en cisaillement sont fondamentales car ce sont elles qui contrôlent la réponse mécanique du matériau, en particulier dans le cas non drainé où, en fonction de la nature dilatante ou contractante des déformations volumiques irréversibles, on observera des réponses très différentes.

La contractance est la composante irréversible (plastique, dans un schéma élastoplastique) de la diminution de volume du sol soumis au cisaillement, et cor- respond à un mécanisme de densification de l'empilement granulaire résultant des glissements et roulements entre grains occasionnés par la sollicitation appliquée.

Inversement, la dilatance est la composante irréversible de l'augmentation de volume du sol soumis au cisaillement, et correspond à un mécanisme de désenchevêtrement et de foisonnement de l'empilement granulaire.

Ces variations de volume, contractantes ou dilatantes, s'ajoutent aux variations de volume réversibles (élastiques), induites par la sollicitation, et l'on adopte généralement, dans le cadre de l'élastoplasticité, la décomposition de la déformation volumique totale en la somme d'une composante élastique et d'une composante plastique:

$$
\varepsilon_{\mathrm{v}}=\varepsilon_{\mathrm{v}}^{\mathrm{e}}+\varepsilon_{\mathrm{v}}^{\mathrm{p}}
$$

Dans le cas non drainé parfait $\left(\varepsilon_{\mathrm{v}}=0\right)$, les comportements contractant et dilatant se traduisent par un taux de développement des surpressions interstitielles d'origine "canélastique " positif et négatif respectivement, pouvant conduire à des surpressions positives très élevées dans le cas contractant et fortement négatives dans le cas dilatant.

Le caractère contractant ou dilatant du comportement volumique d'un sable est directement lié à l'état initial du matériau, exprimé principalement en termes d'indice des vides e (à relier à l'indice de densité $\left.I_{D}=\left(e_{\text {max }}-e\right) /\left(e_{\text {max }}-e_{\text {min }}\right)\right)$ et d'état de contrainte initial applique (état de consolidation).

\section{2}

\section{Cisaillement drainé et non drainé d'un sable à l'appareil triaxial}

La figure 2 présente les comportements typiques observables à l'appareil triaxial lors du cisaillement drainé d'un sable à partir d'un état initial lâche et dense (pour un même niveau de consolidation isotrope appliqué). Les résultats sont présentés dans les plans $\left(q, \varepsilon_{\mathrm{a}}\right)$ pour les courbes de cisaillement, ( $\Delta V / N$ et $\varepsilon, \varepsilon$ ) pour les variations de volume des éprouvettes et (q, $p$ ) pour les chemins des contraintes, q étant le déviateur des contraintes, p' la contrainte moyenne effective et $\varepsilon_{\mathrm{a}}$ la déformation axiale.

En termes de courbe de cisaillement, on observe une réponse plus rigide pour le sable dense, avec passage par un maximum de résistance au cisaillement, puis radoucissement avec stabilisation progressive vers la même résistance ultime que celle obtenue pour le sable lâche (état de plasticité parfaite, appelé état critique, matérialisé par la ligne LCR sur la figure 2). En termes de déformations volumiques, le sable lâche est uniquement contractant, alors que le sable dense devient très rapidement dilatant, après une phase initiale de contractance.

Le passage de la phase de contractance à la phase de dilatance correspond à un état particulier important, qui définit, dans le plan des contraintes, un seuil de transition linéaire, appelé seuil ou état caractéristique (Luong, 1980), matérialisé par la ligne LCA sur la figure 2, pratiquement confondue avec la ligne LCR, délimitant un domaine sub-caractéristique où le matériau est contractant et un domaine sur-caractéristique où le matériau est dilatant. Dans les deux cas (lâche et dense), on observe finalement une stabilisation progressive du volume vers les grandes déformations, correspondant, dans ce plan 


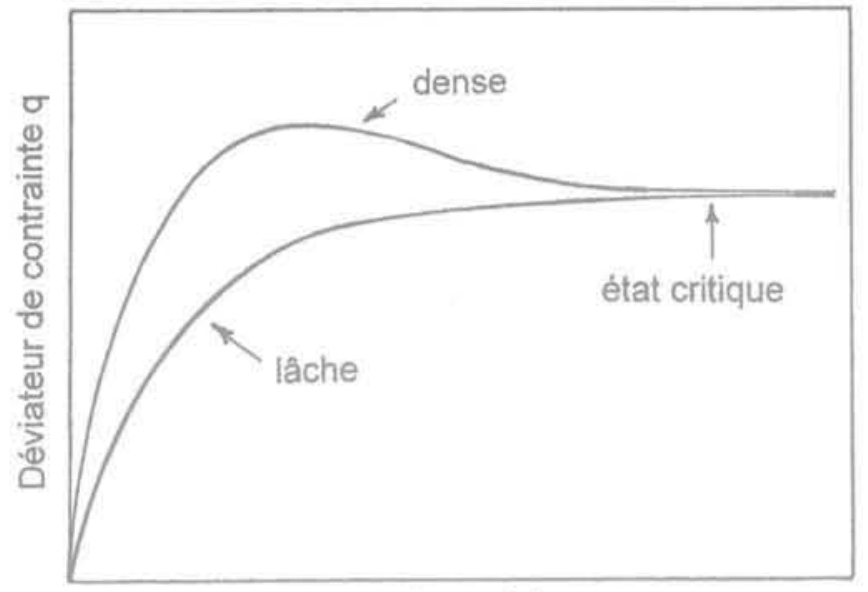

Déformation axiale $\varepsilon_{a}$

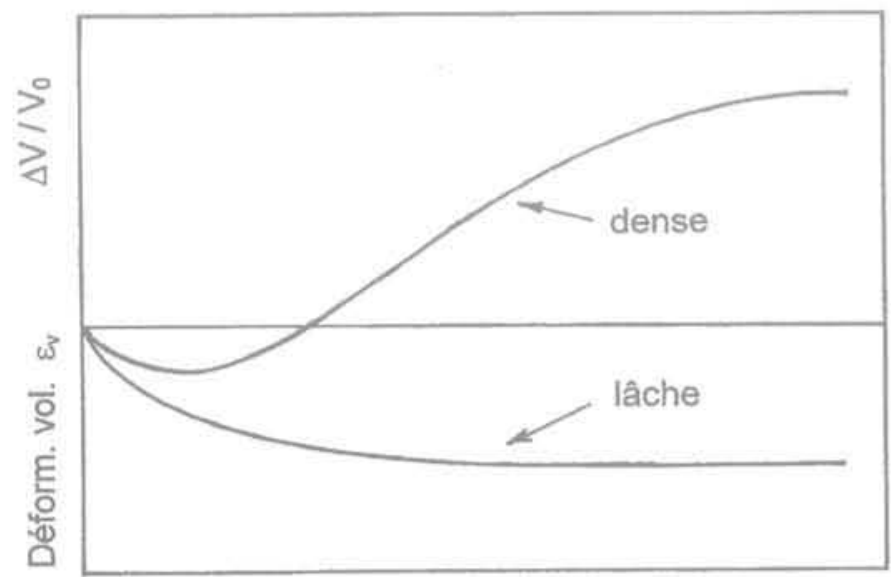

Déformation axiale $\varepsilon_{a}$

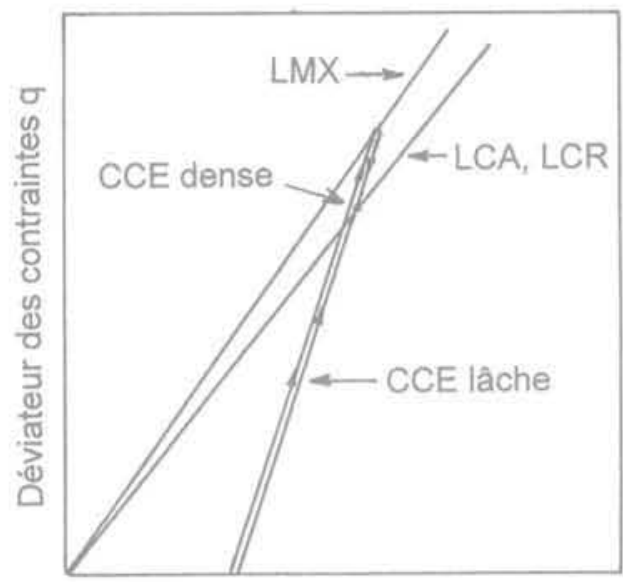

Contrainte moyenne effective $p^{\prime}$

FIG. 2 Comportements typiques observés lors du cisaillement drainé d'un sable en compression à l'appareil triaxial (schématique).

Typical behaviours observed upon drained shear of a sand in compression in the triaxial apparatus (schematic).

aussi, à l'approche de l'état critique, Le plan (q, p') permet, quant à lui, de représenter les chemins des contraintes effectives suivis pendant le cisaillement (CCE), qui sont, dans le cas drainé, des segments de droite, inclinés à 3 pour 1 sur l'horizontale. On note que les inclinaisons des contraintes $\eta\left(\eta=q / p^{\prime}\right)$ correspondant à l'état caractéristique $\left(\eta_{\mathrm{CA}}\right)$ et à l'état critique $\left(\eta_{\mathrm{CB}}\right.$ ou $\mathrm{M}$ ) sont très proches l'une de l'autre et seront

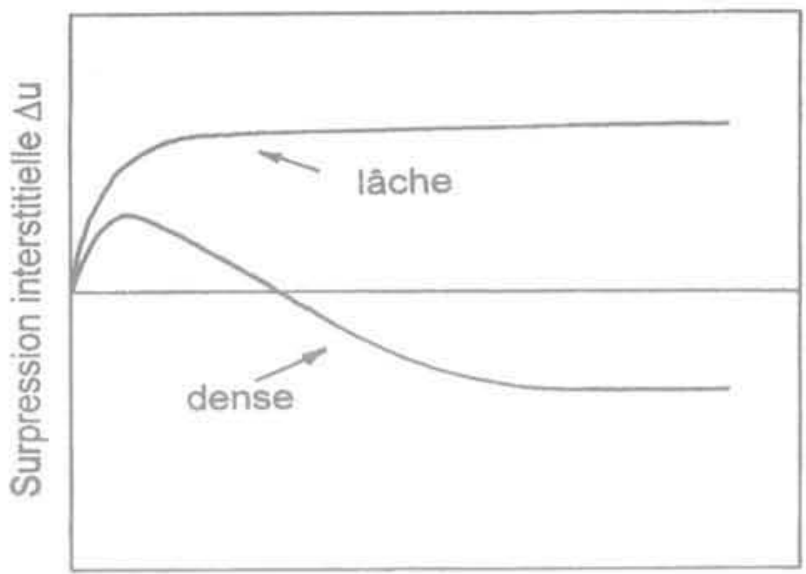

Déformation axiale $\varepsilon_{\mathrm{a}}$

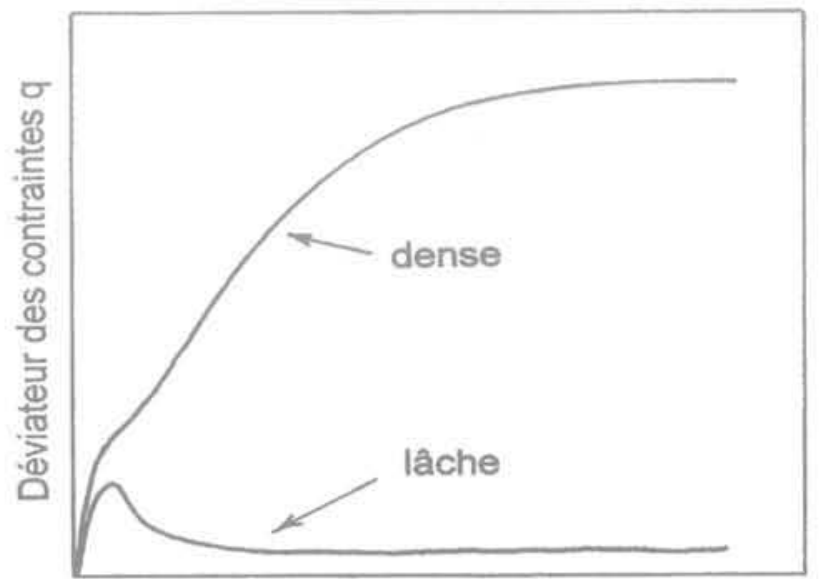

Déformation axiale $\varepsilon_{\mathrm{a}}$

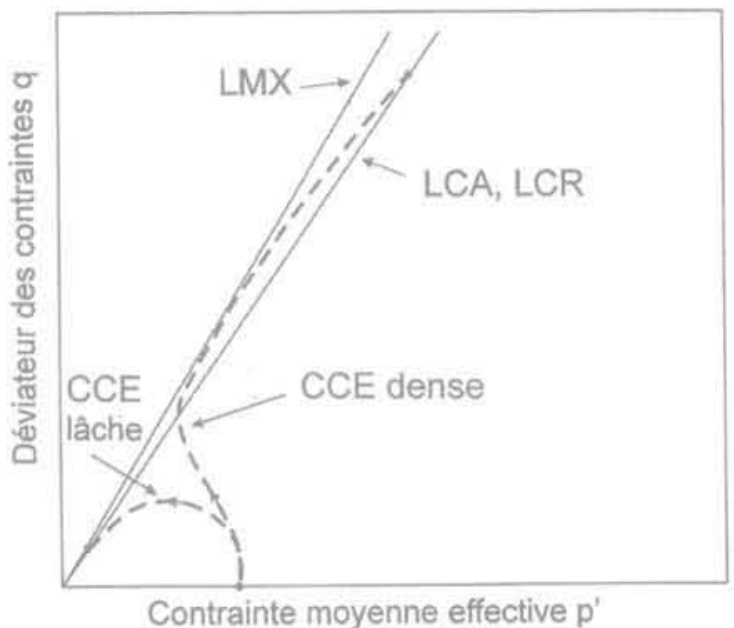

FGG.3 Comportements typiques observés lors du cisaillement non drainé d'un sable en compression à l'appareil triaxial (schématique)

Typical behaviours observed upon undrained shear of a sand in compression in the triaxial apparatus (schematic).

confondues dans la pratique. L'inclinaison des contraintes correspondant à la rupture au maximum de résistance pour le sable dense est notée $\eta_{\mathrm{F}}$ (droite de rupture LMX sur la figure 2).

La figure 3 présente, pour le même matériau et les mêmes conditions initiales que celles de la figure 2, les comportements non drainés correspondants. On constate que le non-drainage (ou condition de volume 
constant) entraîne des réponses totalement différentes de celles de la figure 2 en termes de courbes de cisaillement, avec un comportement très stable, continûment durcissant, pour le sable dense. Pour le sable lâche, par contre, on observe un comportement apparemment très instable, avec un pic très marqué de résistance au cisaillement non drainé, de très faible niveau, suivi d'une chute très rapide de résistance jusqu'à obtention d'une résistance ultime extrêmement faible et pratiquement constante. En termes d'évolution des surpressions interstitielles au cours du cisaillement, le sable lâche a un comportement uniquement contractant, avec un taux de génération de la surpression toujours positif et stabilisation progressive, alors que le sable dense présente tout d'abord une phase contractante à taux positif suivie d'une phase dilatante, à taux négatif, le point à taux nul correspondant à la transition entre contractance et dilatance, où l'on retrouve la notion de seuil caractéristique. Dans le plan (q, p et p'), on définit maintenant pour chaque essai un chemin des contraintes effectives (CCE) distinct du chemin des contraintes totales (CCT). Pour le sable dense, le chemin des contraintes effectives présente un point ( anguleux $)$ typique, plus ou moins marqué, avec remontée du chemin vers la droite de rupture, le point anguleux correspondant pratiquement au franchissement du seuil caractéristique. Pour le sable lâche, par contre, on observe une diminution continue de la contrainte moyenne effective $\mathrm{p}^{\prime}$, avec migration de l'état de contrainte vers les faibles valeurs de q et de p'.

Les résultats typiques présentés sur les figures 2 et 3 montrent déjà que le seul type de comportement qui pourra être à l'origine d'un écoulement déclenché par une sollicitation monotone, qui nécessite une résistance ultime très faible, est celui du sable lâche en conditions non drainées, que l'on va décrire plus précisément cidessous.

\section{3}

\section{Liquéfaction statique des sables lâches}

La thèse de Castro (1969), réalisée au sein de l'équipe de Casagrande (1975), constitue le premier travail de référence qui a permis d'identifier clairement le phénomène de liquéfaction statique, appelée «statique » par abus de langage, puisqu'il s'agit en fait de liquéfaction sous un chargement monotone, qui pourrait être quasi statique ou dynamique. Les recherches dans le domaine se sont ensuite poursuivies aux ÉtatsUnis et au Canada. En France, on peut citer les travaux de P. Habib (1977), de Flavigny et Foray (1986) ainsi que les travaux développés en liaison avec le glissement du nouveau port de Nice de 1979 (Schlosser, 1985; Blondeau, 1986) et les recherches associées, à partir de 1985 (Canou, 1987 ; Canou, 1989). Depuis, les recherches sur la liquéfaction statique se sont considérablement développées au plan français et européen au travers, en particulier, du réseau français du GRECO-Géomatériaux et du réseau européen de laboratoires ALERT.

Bien que fortement mobilisés par les problèmes de liquéfaction des sables sous séisme, les Japonais ne se sont intéressés qu'assez tard à la liquéfaction statique des sables, avec la thèse de Verdugo (1992) réalisée au sein de l'équipe d'Ishihara (1993). Depuis, une activité de recherche importante est consacrée, au Japon, ainsi que, de manière plus générale, au plan international, à l'étude de ce phénomène et de ses conséquences.

\section{1}

\section{Description du phénomène de liquéfaction statique}

La figure 4 montre un résultat typique de liquéfaction statique obtenu dans un essai triaxial de compression réalisé sur le sable d'Hostun RF dans un état très lâche (Canou et al., 1991), présenté dans les plans définis au paragraphe 2.2. Comme mentionné précédemment à partir du schéma de la figure 3 , la courbe de cisaillement présentée dans le plan $(q, \varepsilon)$ de la figure 4 se caractérise par un pic de résistance au cisaillement très marqué et de faible niveau, qui se manifeste pour une déformation axiale faible (un peu moins de $1 \%$ ), suivi d'une chute très rapide de résistance avec stabilisation progressive autour d'une valeur ultime constante très faible. Le phénornène de liquéfaction concerne toute la partie radoucissante du comportement, depuis l'initiation (au pic), jusqu'à l'état ultime « liquéfié » correspondant à l'état du matériau atteint dans un écoulement. L'évolution de la surpression interstitielle développée lors du cisaillement rend compte du caractère très contractant du sable, avec un taux de croissance très fort dès le début de l'essai et stabilisation progressive de la surpression vers une valeur résiduelle
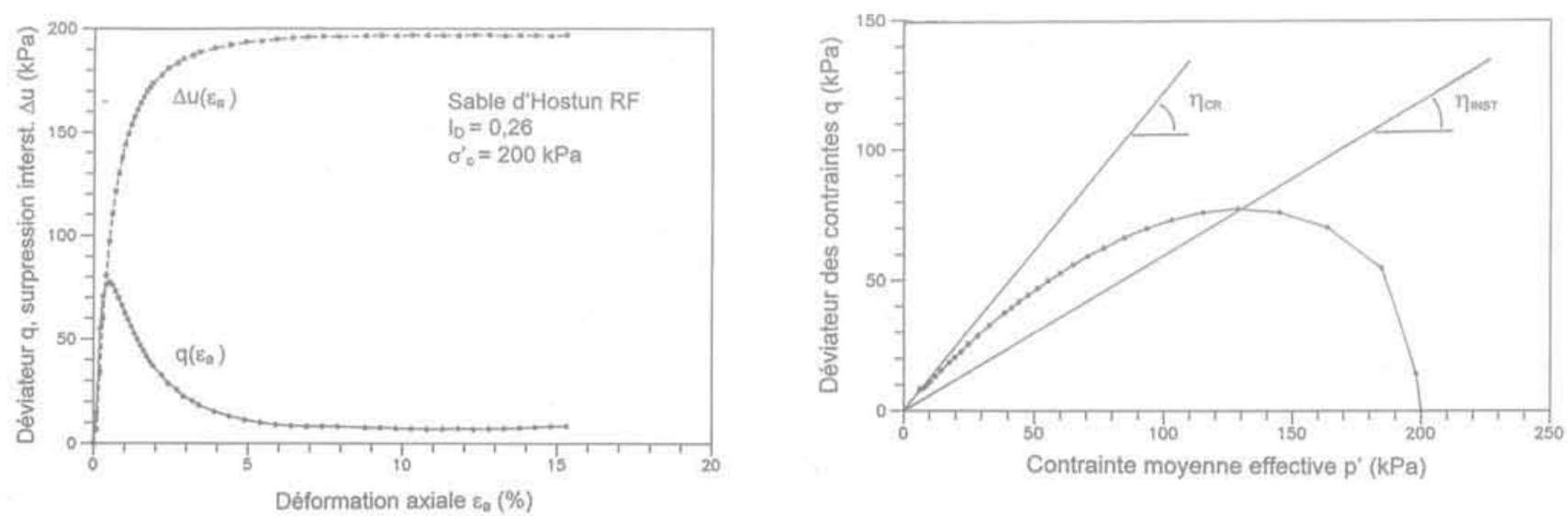

FG. 4 Essai de liquéfaction statique typique réalisé à l'appareil triaxial (Canou et al., 1991).

Typical static liquefaction test carried out in the triaxial apparatus (Canou et al., 1991). 
Iorsque l'on va vers les grandes déformations. Cette stabilisation est à associer à la stabilisation du déviateur des contraintes dans le plan ( $q, \varepsilon_{\mathrm{a}}$ ). Le plan (q, p') présente la forme typique du chemin des contraintes effectives suivi au cours de l'essai, avec une décroissance continue de la contrainte effective moyenne au cours du cisaillement et migration progressive de l'état des contraintes effectives (q, p') vers l'origine avec stabilisation, à l'état ultime, sur un point d'accumulation, après passage par le pic de résistance.

L'inclinaison des contraintes $\eta$ définie précédemment rend compte du niveau de frottement mobilisé au cours du cisaillement $\left(\eta=6 \sin \phi_{\text {mot }} /\left(3-\sin \phi_{\text {mat }}\right)\right.$ pour les essais de compression). Ce paramètre varie entre zéro (état de contrainte initial isotrope) et $\eta_{F}$ pour la rupture de Mohr-Coulomb classique, définie au maximum de. résistance au cisaillement dans un essai drainé. Le chemin des contraintes de la figure 4 montre que l'inclinaison des contraintes $\eta_{\text {INS }}$ au pic de résistance correspond à un angle de frottement mobilisé sensiblement plus faible que l'angle de frottement mobilisé à l'état ultime, qui correspond, lui, à la rupture de Mohr-Coulomb classique, qui, dans le cas du sable lâche, correspond aussi à l'état critique du matériau $\left(\eta_{F}=\eta_{C_{R}}=M\right)$. Ceci montre donc que, bien que l'on ait toujours un écrouissage positif du matériau au sens de la mobilisation du frottement, qui continue de croître entre $\phi_{\text {INST }}$ et $\phi_{\text {f }}^{\prime}$, on observe une chute très forte de résistance au cisaillement, montrant que l'on a affaire à une instabilité particulière, qui n'a rien à voir avec la rupture classique de Mohr-Coulomb, que l'on retrouve ici à l'état ultime. D'où des approches du phénomène du type instabilité qui se sont développées dans la littérature pour décrire le phénomène et sur lesquelles on revient dans le paragraphe 3.2.

\section{2}

\section{Influence de différents paramètres sur le phénomène}

L'initiation et le développement du phénomène de liquéfaction statique sont très sensibles à de nombreux facteurs parmi lesquels on trouve tout d'abord la nature du sable (matériau constitutif des grains, caractéristiques granulométriques, angularité et état de surface; présence de fines, etc.), l'état initial du matériau, en

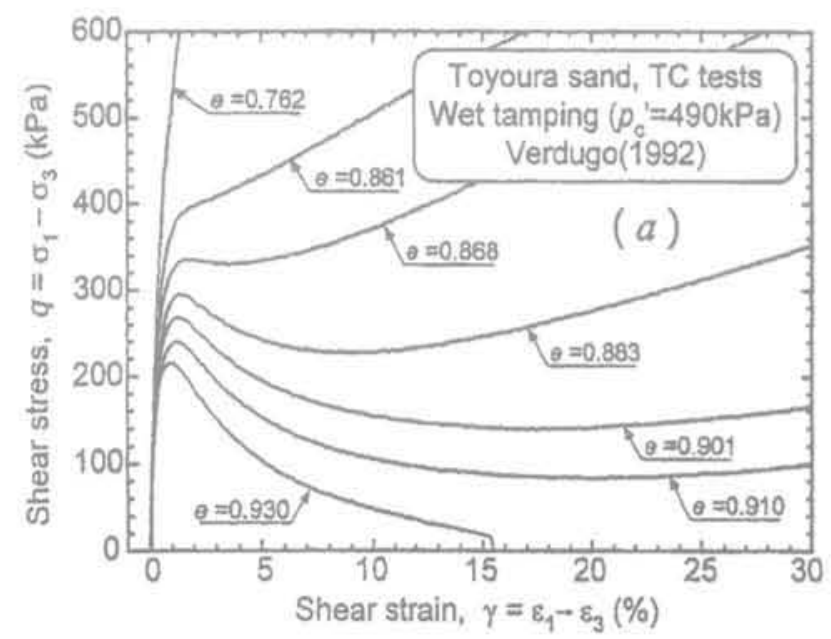

termes d'indice des vides, de structure (arrangement des grains), d'anisotropie, de qualité de saturation, d'état de contrainte initial et finalement les caractéristiques de la sollicitation appliquée (chemin de contrainte ou de déformation suivi, vitesse de sollicitation, etc.). Il convient de rajouter qu'en situation réelle, sur site, la prévision du déclenchement et du développement d'un écoulement de liquéfaction est fortement compliquée par le fait que les facteurs mentionnés cidessus et que l'on peut contrôler de manière raisonnable au laboratoire sont beaucoup plus difficiles à évaluer, à quoi se rajoutent les caractéristiques de drainage, les conditions aux limites du problème réel ainsi que des mécanismes d'écoulement souvent complexes (mécanismes souvent régressifs) et difficiles à prévoir. On présente dans la suite l'influence de quelques paramètres significatifs sur l'initiation et le développement du phénomène de liquéfaction statique dans les sables.

\section{$3,2,4$}

\section{Indice des vides}

L'indice des vides (à relier à l'indice de densité) est un paramètre fondamental qui conditionne, pour une grande part, en combinaison avec le niveau de contrainte initial appliqué, le caractère contractant ou dilatant des sables. Pour un sable de caractéristiques données, ce paramètre doit donc a priori avoir une influence déterminante sur l'initiation et le développement du phénomène de liquéfaction statique. Cette influence a été clairement mise en évidence par différents auteurs (entre autres, Castro, 1969; Canou, 1989) et confirmée depuis sur différents sables, montrant que l'on passe de manière continue d'un comportement fortement « liquéfiant » pour les structures très lâches à un comportement très stable de sable dense pour les faibles indices des vides. On peut même, pour les structures les plus lâches, obtenir une liquéfaction totale du matériau, au sens de l'annulation des contraintes effectives (résistance ultime nulle) avec effondrement soudain, très caractéristique, de l'éprouvette à l'appareil triaxial, ce qui permet de définir un indice des vides de liquéfaction totale e $e_{\text {eff }}$ (Canou, 1989: Canou et al., 1990). La figure 5 montre un ensemble de résultats obtenus plus récemment (Verdugo, 1992) qui confirme le passage continu du comportement « liquéfiant ») au com-

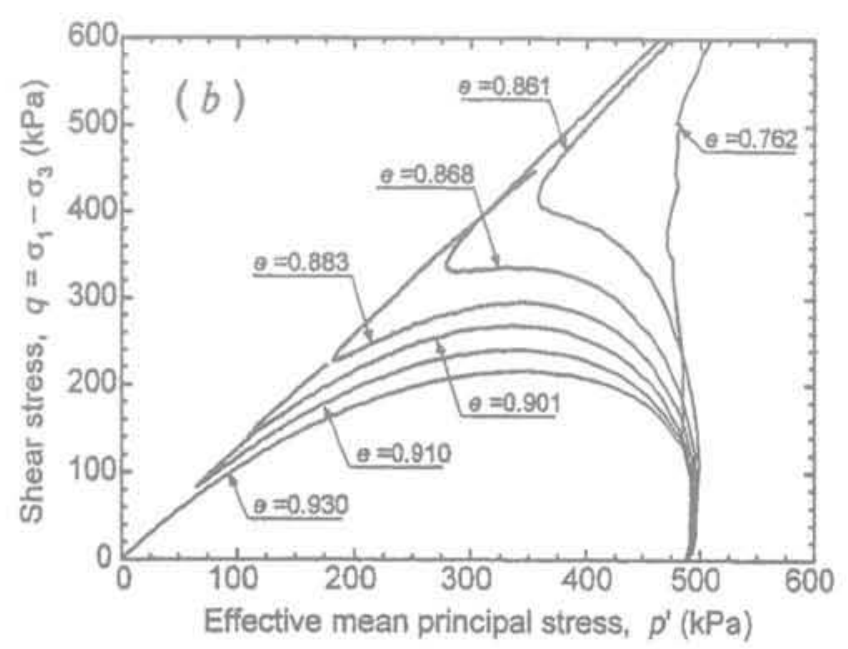

FIG.5 Influence de l'indice des vides sur les comportements observés à l'appareil triaxial (Verdugo, 1992). Influence of void ratio on the behaviours observed in the triaxial apparatus (Verdugo, 1992). 
portement durcissant. Lorsque l'état initial de densité du sable augmente, l'état ultime " parfaitement ") constant et de très faible niveau évolue vers un minimum de résistance, suivi d'une phase de durcissement de dilatance, d'autant plus marquée que le sable est dense. Le point pour lequel le minimum de résistance est obtenu correspond au passage entre contractance et dilatance, et donc à l'état caractéristique, que l'on retrouve au point anguleux du chemin des contraintes dans le plan (q, p'). On a donc coexistence, sur une certaine plage d'états initiaux, entre une phase de radoucissement initiale post-pic (amorce de liquéfaction) suivie d'une phase de durcissement. Lorsque la partie radoucissante est de faible ampleur (typiquement cas de l'essai à e =0,883 sur la figure 5), on parle de liquéfaction limitée (Castro, 1969; Vaid and Chern, 1985). Dans ce cas, si la résistance de pic est franchie dans un essai piloté en effort, une instabilité va alors se développer avec accumulation rapide de déformations, mais le matériau se stabilisera de nouveau dans la phase dilatante lorsque la résistance mobilisable sera à nouveau égale à la résistance de pic (pour une déformation de l'ordre de $20 \%$ dans l'essai mentionné ci-dessus). Concrètement, dans le cas d'un problème de pente, l'amorce d'un écoulement pourra alors être « bloquée » par dilatance alors que l'écoulement se développera librement dans le cas d'une résistance ultime faible.

\section{$3: 9.8$}

\section{Niveau de consolidation isotrope}

L'augmentation du niveau de consolidation isotrope $\sigma_{c}^{\prime}$ ou $p_{c}^{\prime}$ appliqué à un sable augmente, de manière générale, d'une part, son caractère contractant, et, d'autre part, ses ( performances ) mécaniques (caractéristiques d'élasticité, niveaux de résistance au cisaillement atteints à la rupture). La figure 6 montre qué, pour une plage de contraintes de consolidation suffisamment large, on peut même passer d'un comportement dilatant durcissant (faibles valeurs de $p_{\text {. }}^{\prime}$ ) à un comportement contractant liquéfiant (valeurs élevées de $\mathrm{p}_{\mathrm{c}}$ ). D'autres résultats, obtenus dans le domaine du comportement « liquéfiant ) uniquement (Canou, 1989) montrent que le pic de résistance où est initié le mécanisme de liquéfaction est pratiquement proportionnel à $p_{c}$.' L'augmentation du niveau de consolidation isotrope a donc pour effet de stabiliser le matériau vis-àvis de la liquéfaction, dans le sens où elle augmente l'amplitude seuil de la sollicitation nécessaire pour initier l'instabilité. Par contre, si le seuil d'initiation est franchi, la chute de résistance qui s'ensuivra sera d'autant plus importante et brutale que ce seuil est élevé et l'écoulement se développera avec des conséquences d'autant plus graves.

\section{3}

\section{Influence de l'anisotropie de consolidation}

Dans la nature, les sols ne sont généralement pas consolidés de manière isotrope et sont soumis à un cisaillement initial, plus ou moins important en fonction de la configuration (état des terres au repos $\mathrm{K}_{0}$ présence de déclivités, etc.). Il est donc important de pouvoir évaluer l'influence de ce paramètre sur les caractéristiques de liquéfaction d'un sable. Castro (1969) montre déjà, de manière ponctuelle, que l'initiation de la liquéfaction semble être favorisée par l'existence d'un déviateur de contrainte initial. L'influence très défavorable de ce paramètre est clairement mise en évidence par les travaux de Seed (1983) menés en liaison avec les sinistres des ports de Nice et de Dunkerque (Schlosser, 1985; Blondeau, 1986), de Kramer and Seed (1988) et de Canou (1989) menés sur des sables différents. Pour des rap-

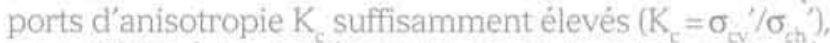
on a affaire à un matériau tellement instable qu'il présente, dès le début du cisaillement non drainé, un comportement radoucissant avec développement de la liquéfaction sans être capable de mobiliser de résistance au cisaillement non drainé supplémentaire (Fig. 7, Canou et al., 1991). Des résultats similaires ont été obtenus dans le cadre de travaux plus récents (Matiotti et al., 1996), confirmant l'influence très défavorable d'un cisaillement initial sur l'initiation du phénomène.
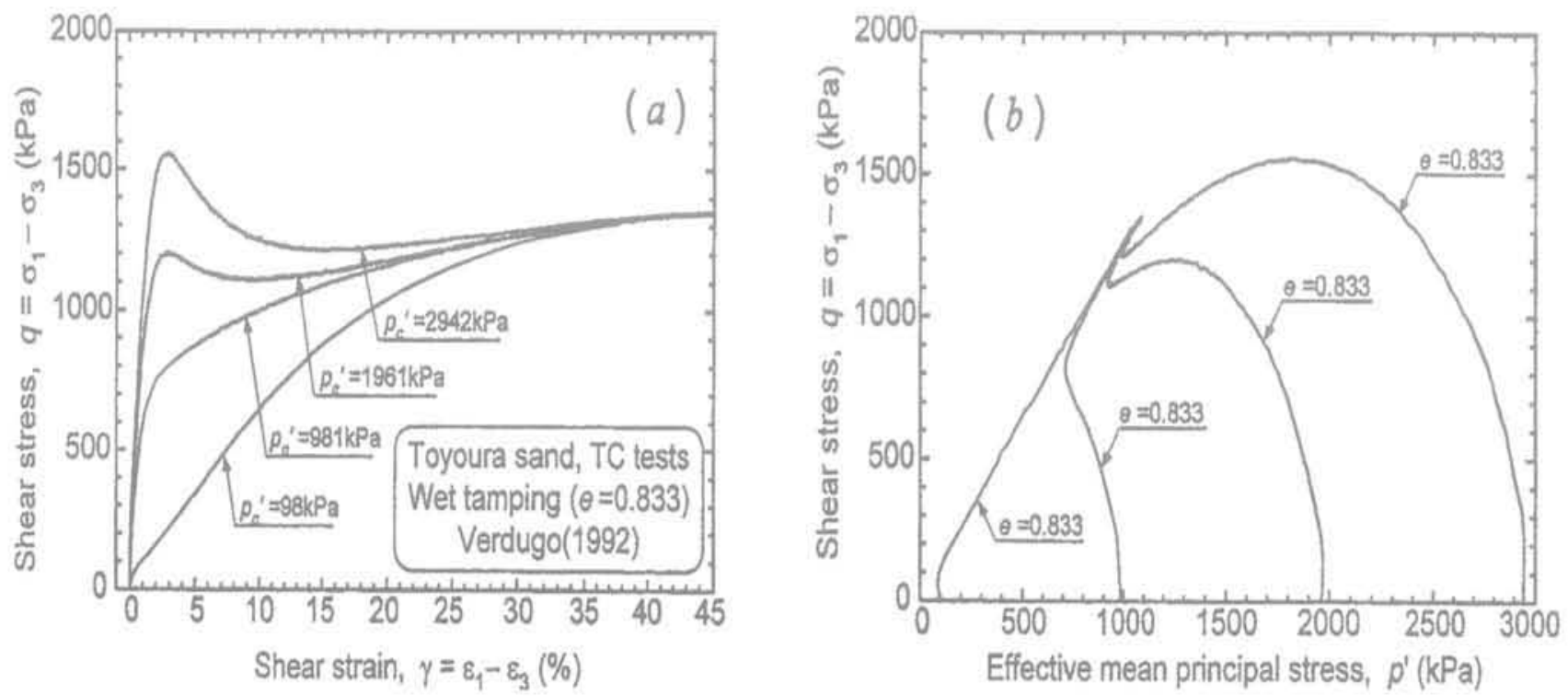

FG.6 Influence du niveau de consolidation isotrope sur les comportements observés à l'appareil triaxial (Verdugo, 1992).

influence of the level of isotropic consolidation on the behaviours observed in the triaxial apparatus (Verdugo, 1992). 

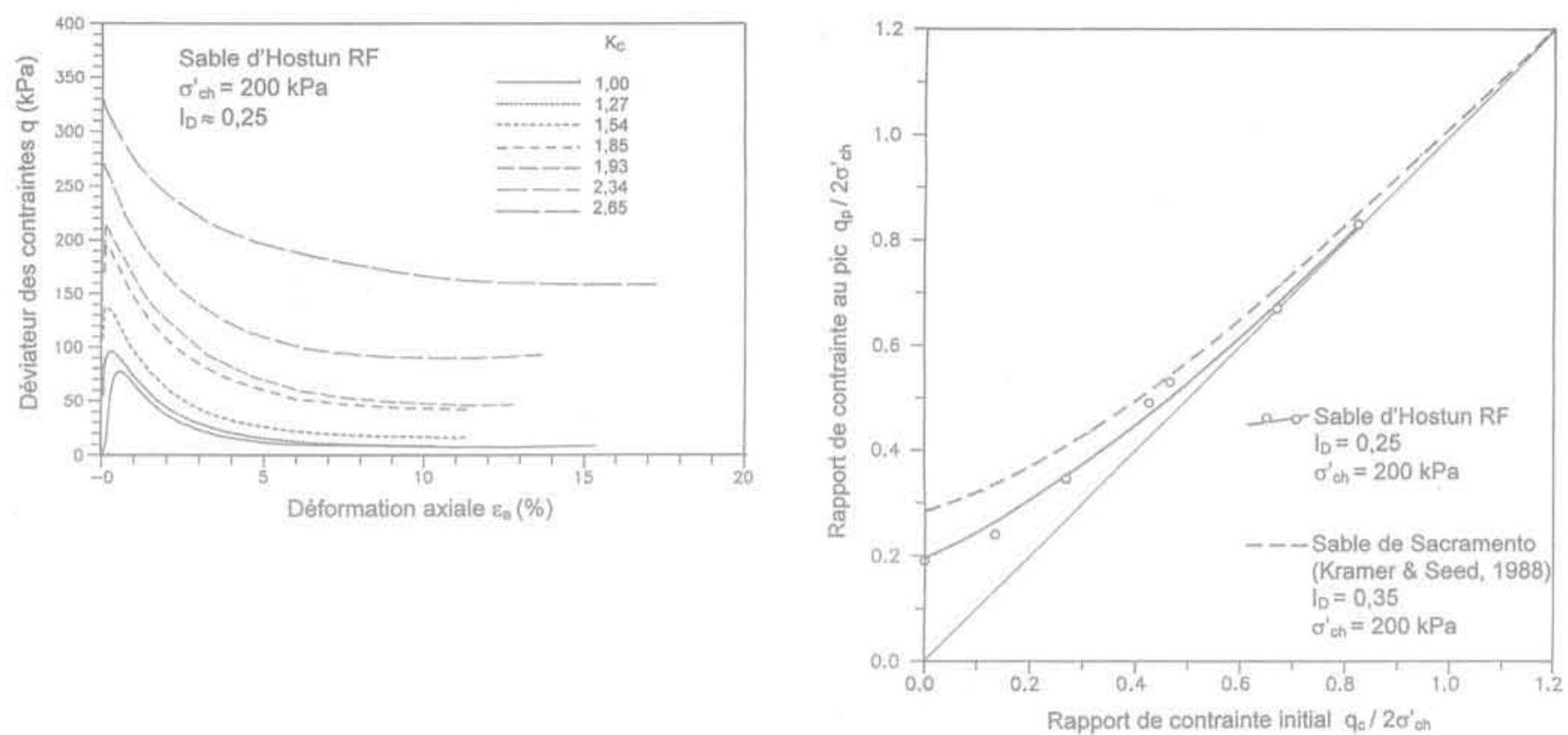

FiG.7 Influence de l'anisotropie de consolidation sur l'initiation et le développement de la liquéfaction statique (Canou et al., 1991).

Influence of the anisotropy of consolidation on the initiation and development of static liquefaction (Canou et al., 1991).

\section{2 .4}

\section{Trajet de chargement (compression-extension)}

L'appareil triaxial permet d'explorer des chemins de compression et d'extension, au sens des déformations, les contraintes appliquées restant toujours de compression pour les sables. Lors des essais de compression, la contrainte axiale est principale majeure, alors qu'elle devient contrainte principale mineure dans les essais d'extension. On constate généralement une réponse plus faible du matériau lors des sollicitations d'extension, avec un comportement volumique généralement plus contractant en extension qu'en compression (Dupla et al., 1995; Matiotti et al., 1996). On peut même observer, pour certains indices de densité intermédiaires, des différences qualitatives importantes, avec une réponse dilatante durcissante en compression et une réponse contractante de type liquéfaction en extension (De Gennaro et al., 1996; Benahmed, 2001 , Fig. 8). Ces différences observées entre compression et extension prendront toute leur importance lorsque l'on s'intéressera aux sollicitations cycliques alternées pour lesquelles les instabilités et les ruptures seront généralement initiées lors d'une phase d'extension.

\section{2 .5}

\section{Structure initiale}

La structure initiale, au sens de l'arrangement géométrique initial des grains de sable dans l'empilement joue un rôle important sur la réponse mécanique macroscopique du matériau, c'est-à-dire que l'indice des vides et l'indice de densité correspondant ne sont pas suffisants pour caractériser le comportement mécanique et qu'il serait nécessaire d'introduire un paramètre permettant de " quantifier » l'influence de cette structure vis-à-vis de la réponse mécanique du sable. L'influence de la structure sableuse peut être étudiée de manière indirecte au laboratoire en utilisant des pro- cédures de reconstitution des éprouvettes bien différenciées, permettant d'obtenir des arrangements distincts. Pour la reconstitution d'éprouvettes de sable lâche, on peut utiliser, entre autres méthodes, un mode de dépôt de type pluviation à sec et un mode de dépôt par damage humide du matériau. La cohésion capillaire résultant de la faible teneur en eau utilisée dans le deuxième cas favorise la formation de structures lâches. On peut cependant préparer des éprouvettes au même indice des vides par les deux méthodes, les différences éventuelles de comportement observées, rendant alors compte de l'influence de la structure initiale de fabrication sur le comportement (Canou, 1989; Benahmed, 2001). La figure 9 montre que le damage humide favorise une réponse plus contractante que la pluviation à sec et l'on est donc en présence d'un matériau plus «liquéfiable » (Benahmed, 2001). On peut donc parler de structures « stables », plutôt dilatantes, constituées d'empilements réguliers de grains (dépôt $(s e c$ ), et de structures " instables » ou " métastables », contractantes, plus irrégulières, formées d'agrégats et de macro-pores, et susceptibles de s'effondrer.

\section{$3,2.6$}

\section{Autres paramètres}

De nombreux autres paramètres que ceux décrits cidessus, que l'on ne détaillera pas dans ce texte, peuvent avoir une influence significative sur l'initiation et le développement du phénomène de liquéfaction « statique ». En ce qui concerne, par exemple, les caractéristiques granulométriques mentionnées plus haut, les sables fins et uniformes à grains arrondis sont généralement considérés comme plus liquéfiables que les sables grossiers à granulométrie étalée et à grains anguleux. De même, la présence d'un faible pourcentage de fines au sein de la matrice sableuse peut favoriser la contractance et, donc, un comportement « liquéfiant ». L'anisotropie inhérente ou initiale (résultante 

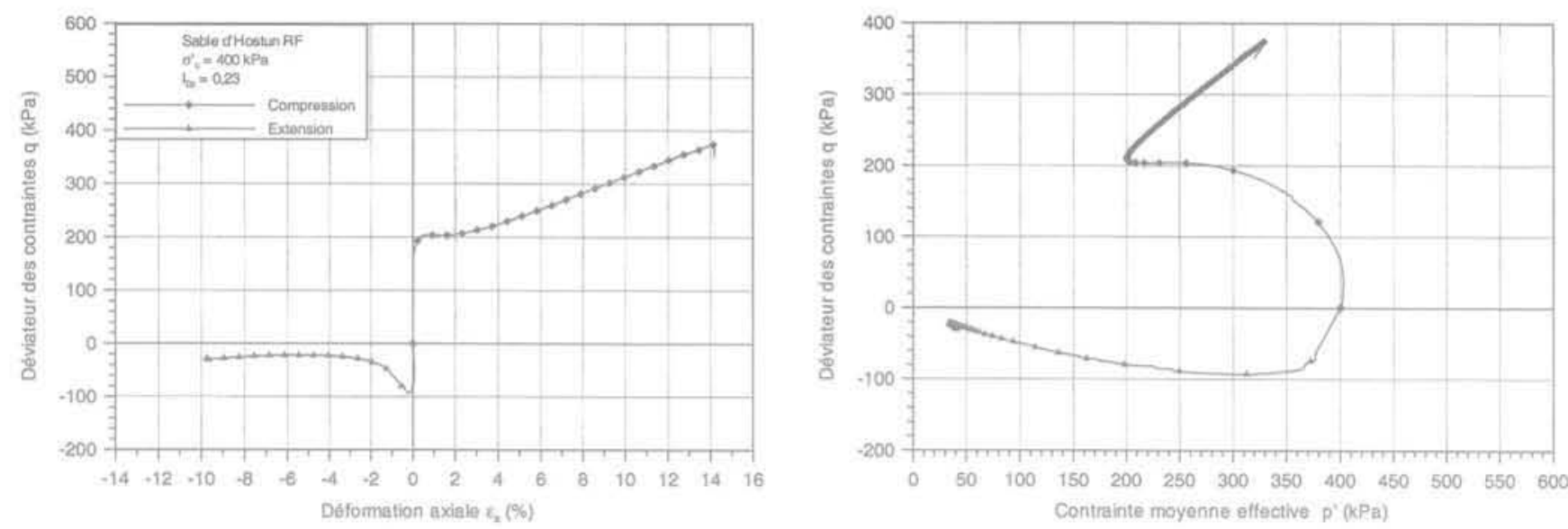

FG.8 Influence du trajet de chargement (compression-extension) sur les comportements observés (Benahmed, 2001). Influence of loading path (compression-extension) of the behaviours observed (Benahmed, 2001).
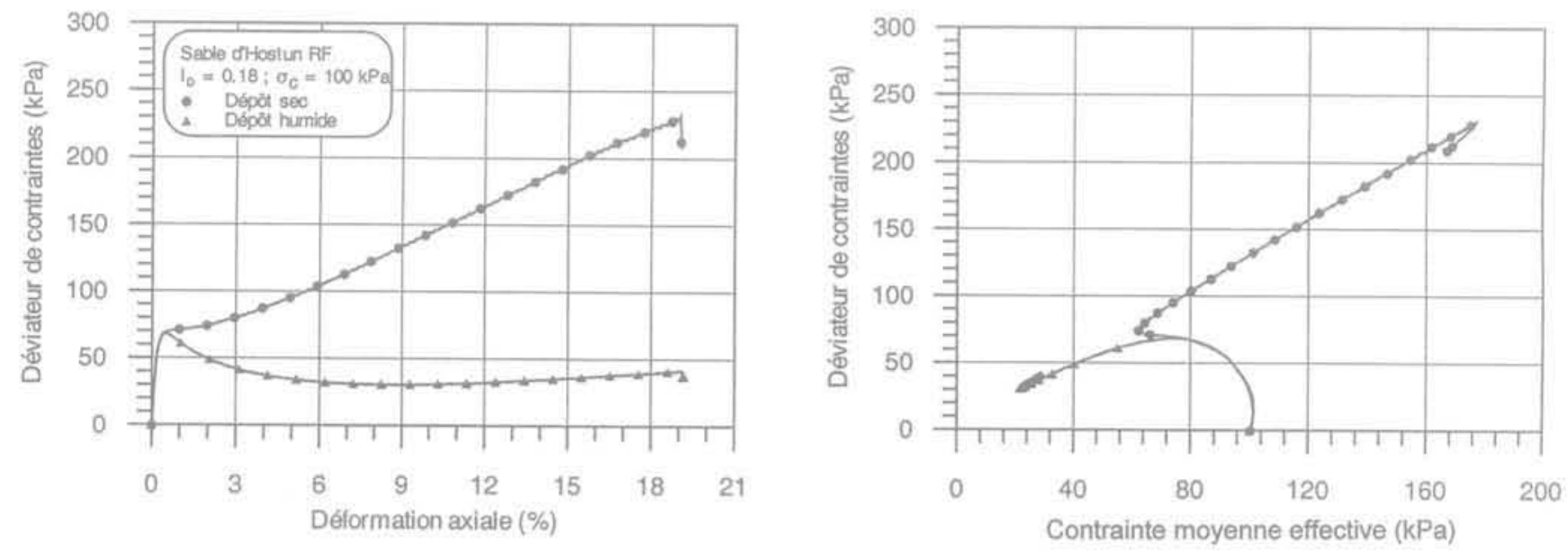

FG.9 Influence de la structure sableuse initiale sur les comportements observés (Benahmed, 2001). Influence of initial sand structure on the behaviours observed (Benahmed, 2001).

complexe des caractéristiques granulaires, du mode de fabrication ou de formation, de l'état de contrainte initial appliqué, etc.), généralement isotropie transverse, qui peut entraîner des contrastes importants de propriétés mécaniques dans les sols selon la direction de sollicitation, peut avoir une influence sur sa \& liquéfiabilité », en combinaison avec le chemin de contrainte ou de déformation appliqué (compression-extension, influence de la contrainte intermédiaire, rotation des axes principaux de contrainte, etc.).

\section{3}

\section{Initiation de l'instabilité de liquéfaction}

Le phénomène de liquéfaction sous chargement monotone est initié au pic de résistance au cisaillement, au-delà duquel la chute de résistance est amorcée avec développement rapide de grandes déformations, lorsque la sollicitation est appliquée en effort, jusqu'à obtention de l'état « ultime » pour lequel le matériau peut s'écouler avec une résistance ultime très faible. Il est donc important de s'attacher à caractériser et « quantifier », dans la mesure du possible, les conditions d'initiation de l'instabilité de liquéfaction, condition nécessaire au développement d'un éventuel écoule- ment. A partir d'un ensemble d'observations expérimentales, Sladen et al. (1985) proposent le concept de surface d'effondrement, correspondant au lieu des points, dans l'espace (q, p', e) où l'instabilité de liquéfaction est initiée. Ces auteurs observent que, lorsque l'on réalise des essais de liquéfaction statique sur un sable donné, à différents niveaux de consolidation isotrope et un même indice des vides a consolidé $n$ des éprouvettes, les pics de résistance sont alignés dans le plan $\left(q, p^{\prime}\right)$ et la droite qui les joint passe par le point d'état ultime (état critique ou état stable, Fig. 10a). Lorsque l'indice des vides varie, on obtient un faisceau de droites parallèles entre elles, s'appuyant sur les états ultimes respectifs, ce qui génère dans l'espace (q, p', e) une surface cylindrique, la surface d'effondrement, s'appuyant sur la ligne d'état critique et de génératrices l'ensemble des droites d'effondrement (Fig. 10b). L'intérêt de la surface d'effondrement est de pouvoir caractériser le lieu des états du matériau ou va s'initier la liquéfaction et constitue donc un outil intéressant en vue d'analyses de stabilité de massifs sableux. Le concept n'est cependant utilisable tel quel que sur une plage donnée d'états du matériau, « ni trop lâches, ni trop denses » et doit être adapté en dehors de cette plage (Canou et al., 1990). De même, le lieu des points d'initiation de la liquéfaction peut dépendre de manière 

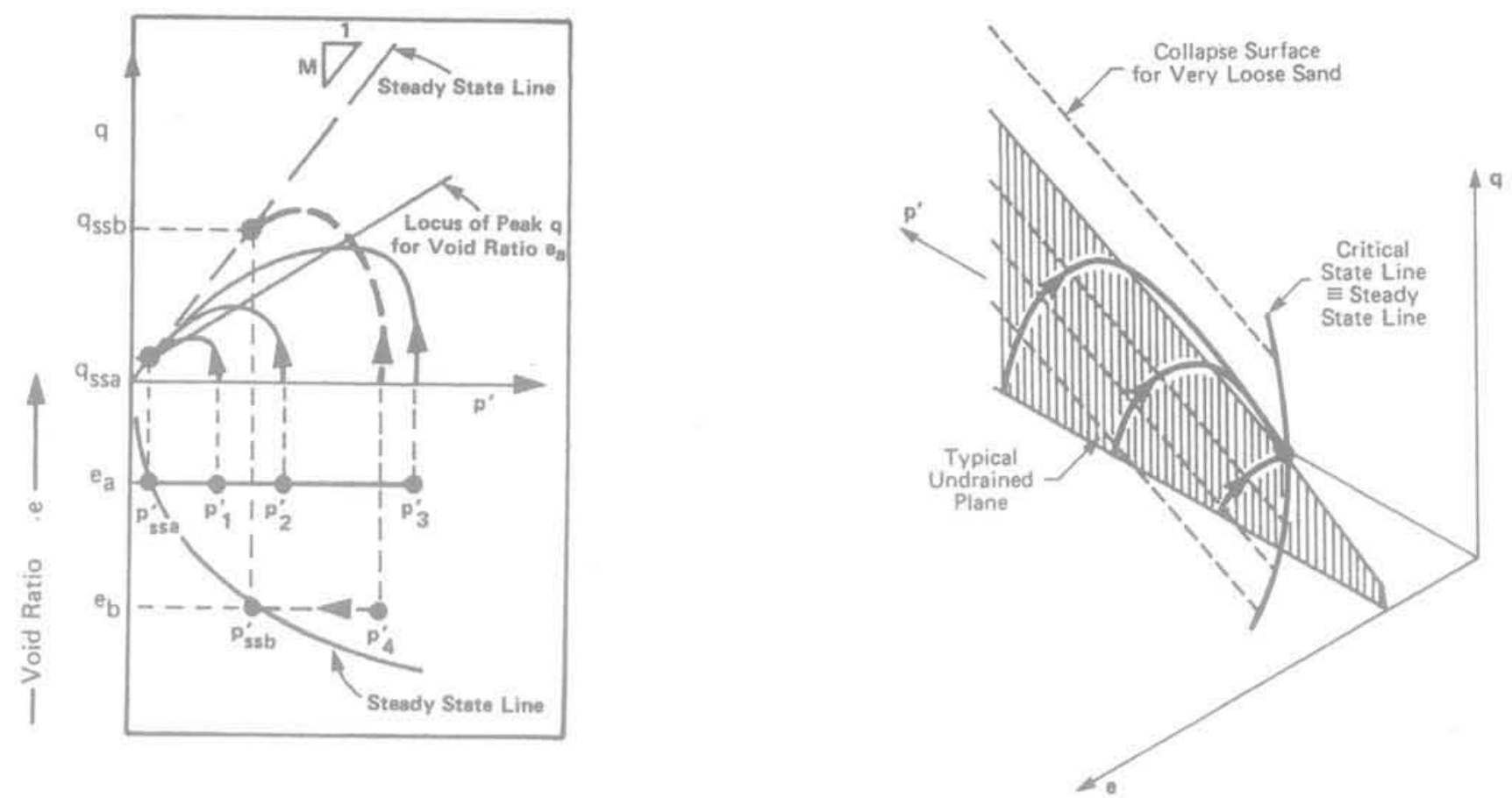

FG. 10 Définition de la droite et de la surface d'effondrement (Sladen et al., 1985).

Definition of the collapse line and the collapse surface (Sladen et al., 1985).

significative de l'histoire antérieure du chargement du sol, en particulier en termes d'état de consolidation du matériau. Pour des états de consolidation fortement anisotropes, par exemple, le concept de surface d'effondrement doit aussi être adapté (Canou et al., 1991).

Lade et son équipe (Lade el al., 1988; Lade and Pradel, 1990) étudient les conditions de stabilité et d'instabilité des sables et montrent que, pour ces matériaux granulaires obéissant à une règle d'écoulement non associée, les conditions d'instabilité de Hill (1958) et de Drucker (1951) basées sur la « négativité » du travail plastique du second ordre ne sont pas suffisantes dans le cas des matériaux dilatants pour assurer l'instabilité (ces matériaux restent en effet particulièrement stables en conditions drainées et non drainées pendant toute la phase de dilatance pré-pic, alors que les critères mentionnés ci-dessus voudraient qu'ils soient instables). Ces auteurs montrent que des instabilités du type liquéfaction ne peuvent apparaitre, à l'intérieur de la surface limite, que dans le cas d'un comportement non drainé (ou à volume constant) et pour un matériau contractant, qui va alors générer des surpressions interstitielles élevées, responsables de l'initiation et du développement du phénomène de liquéfaction (une discussion est menée sur l'influence de la qualité de la saturation du matériau, qui, si elle n'est pas bonne, va affaiblir la condition de non variation de volume du matériau et atténuer, en quelque sorte, le caractère instable du comportement observé (pic moins marqué, ayant tendance à disparaitre)). Lade (1992) introduit la notion de ligne d'instabilité dans le plan $\left(q, p^{\prime}\right)$, lieu des points où est amorcée la liquéfaction. Ce seuil est de mème nature que la droite d'effondrement proposée par Sladen et al. (1985), si ce n'est qu'il passe ici par lorigine des axes (Fig. 11), alors que, pour un indice des vides donné, la droite d'effondrement passe par le point d'état stable correspondant. Vaid and Chern (1985) introduisent, de même, la droite CSR, passant par l'origine des axes, correspondant, elle aussi, au lieu des points où est amorcée la liquéfaction.

Darve et son équipe (Darve, 1993; Mégachou, 1993; Darve et Laouafa, 1999) étudient aussi les conditions d'initiation de l'instabilité de liquéfaction, montrant, eux aussi, que celle-ci ne peut apparaitre que dans le cadre d'une règle d'écoulement non associée. Ces auteurs montrent, en particulier, sur la base de simulations réalisées à partir d'un modèle de comportement incrémental, l'influence d'une variation de volume du milieu (augmentation ou diminution) par rapport à la nonvariation parfaite, sur le comportement observé, Dans le cas d'une diminution de volume représentative d'une certaine compressibilité, simulant, par exemple, une saturation imparfaite du matériau (voir ci-dessus), on observe une " atténuation » du phénomène de liquéfaction, avec un pic de moins en moins marqué, qui peut même disparaitre pour des forts niveaux de compres. sibilité (phénomène observé par exernple dans le cas de sables non saturés ou très mal saturés, pour lesquels on observe une réponse stable du type ( drainée $)$ ).

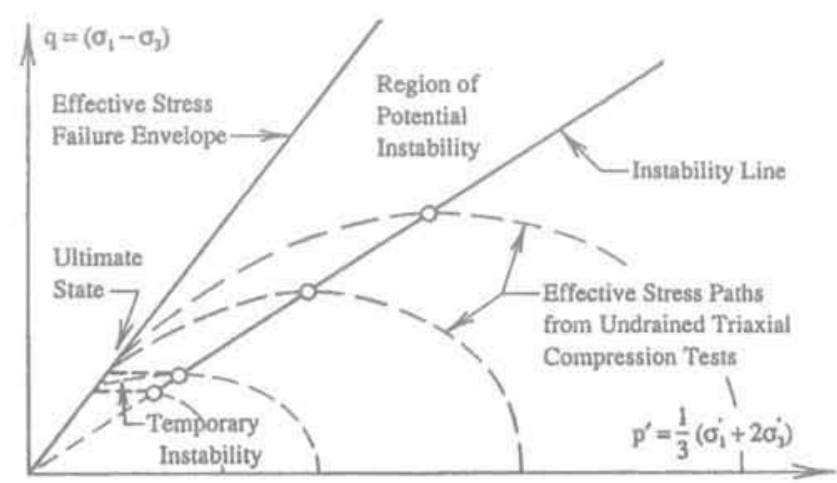

FG.11 Ligne d'instabilité de Lade (1992), Instability line, as defined by Lade (1992). 
Darve et Laouafa (1999) proposent de plus un critère de liquéfaction relativement simple pour les matériaux granulaires dans le cas de configurations axisymétriques, basé sur l'utilisation de l'angle de dilatance $\psi$, ce critère pouvant être généralisé au cas tridimensionnel.

\section{4}

\section{État critique et état stable}

De manière analogue à l'étude des seuils d'initiation du phénomène de liquéfaction sous chargement monotone, il est important de pouvoir caractériser et quantifier l'état ultime du matériau pour lequel la résistance ultime mobilisable en grandes déformations va contrôler l'ampleur de l'écoulement potentiel, dans le cas de problèmes d'écoulement (pentes). Plus cette résistance ultime est faible et plus l'écoulement du type $u$ fluide $)$ sera facilité, avec de grandes distances d'écoulement et des profils de terrain, après restabilisation, à très faible déclivité. De même, plus la différence relative entre la résistance au cisaillement au pic et la résistance ultime est importante et plus le phénomène d'écoulement sera violent, en rapidité et en amplitude. L'observation, pour les états du matériau suffisamment lâches ou contractants, d'états ultimes très particuliers, stabilisés à des niveaux très faibles de résistance (voire résistance ultime nulle pour le cas de la liquéfaction totale), qui peuvent se développer sur une plage de déformation importante (à partir de 3 ou $4 \%$ jusqu'à plus de $20 \%$ de déformation axiale), a conduit les Anglo-Saxons à introduire et développer la notion d'état stable (steady state, introduit à l'origine par Poulos (1981)). On a affaire ici à un cas typique d'écoulement plastique parfait, que l'on observe rarement de manière aussi claire en mécanique des sols, et qui présente, en fait, beaucoup d'analogies avec le concept d'état critique, à la base de la mécanique des sols du même nom, développée par l'école de Cambridge (Roscoe et al., 1958; Schofield and Wroth, 1968). La principale différence mise en avant pour différencier les deux états (état critique et état stable) concerne la formation d'une structure « d'écoulement » (flow structure) qui se développerait au sein du matériau lors des essais de liquéfaction, formation favorisée par des vitesses de déformation élevées, et qui opposerait une résistance au cisaillement minimale à la déformation. Les caractéristiques ultimes correspondant à l'état stable seraient ainsi inférieures à celles correspondant à l'état critique, déterminées à partir d'essais drainés. Les avis sont encore assez partagés sur cette distinction, un argument en faveur de l' " unification » étant de dire que l'état critique, défini par une courbe dans l'espace (q, p', e) matérialisant le lieu de tous les états ultimes du matériau, caractérisés par un comportement plastique parfait, est intrinsèque et qu'il est atteint indifféremment en drainé et en non drainé (liquéfaction pour les sables lâches). Par ailleurs, se pose actuellement de plus en plus le problème, pour un sable donné, de l'unicitê de l'état stable (qui était considéré unique) en fonction de l'état initial du matériau (Konrad, 1990; Konrad et al., 1991; Ibraïm et Doanh, 1997; Benahmed, 2001), et donc, sans doute aussi, de l'état critique, ce qui rajoute encore au débat entre état critique et état stable. La figure 12 montre, dans l'hypothèse d'identité entre état critique et état stable, et d'unicité de cet état, le mécanisme d'obtention de l'état ultime matérialisé par la ligne LCR, en conditions drainées et non drainées, dans les plans ( $q$, p ${ }^{\prime}$ ) et ( $e$, $\mathrm{p}^{\prime}$ ), en fonction de l'état initial (lâche (point A) ou dense (point B)) du matériau.

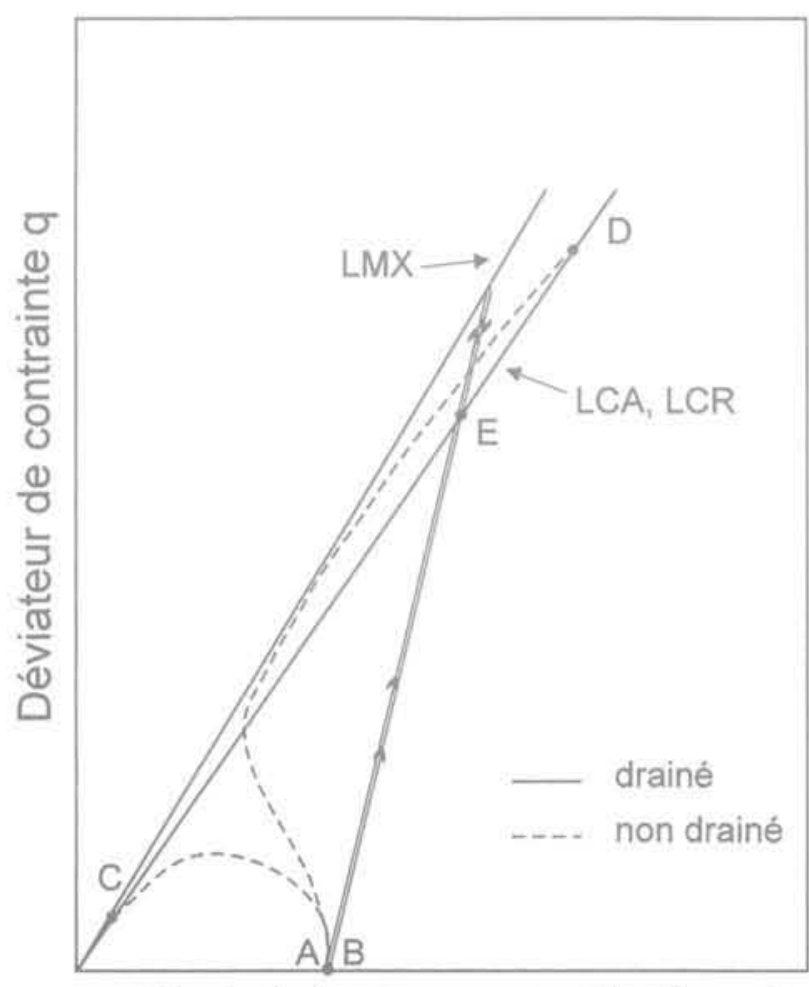

Contrainte moyenne effective $p^{\prime}$

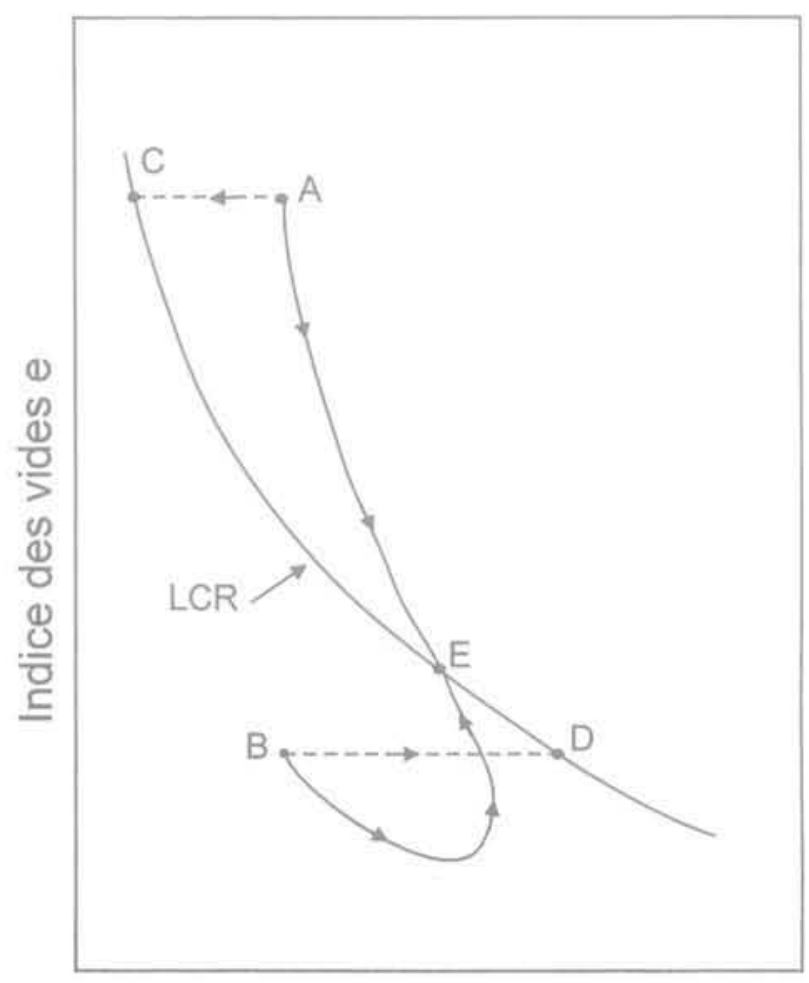

Contrainte moyenne effective $p^{\prime}$

FIG. 12 Chemins drainés et non drainés dans les plans $\left(q, p^{\prime}\right)$ et (e, $\left.p^{\prime}\right)$.

Drained and undrained effective stress paths in the $\left(q, p^{\prime}\right)$ and $\left(e, p^{\prime}\right)$ planes. 


\section{Comportement des sables sous chargement cyclique}

Les sollicitations cycliques appliquées aux sols peuvent être de nature très différentes en fonction de leur origine, en termes d'amplitude, de fréquence, de régularité du signal, etc. Pour ce qui est des séismes, plus particulièrement concernés par les problèmes de liquéfaction, on a affaire à des sollicitations relativement irrégulières, avec cependant, en moyenne, une phase de croissance du niveau de sollicitation jusqu'à une valeur maximale, suivie d'une phase de décroissance jusqu'au retour à l'équilibre. Pour les séismes de magnitude importante dans lesquels sont observés des phénomènes de liquéfaction, les sollicitations sont complexes, tridimensionnelles, avec des amplitudes fortes et des effets dynamiques importants. Pour ces différentes raisons, il est donc en pratique impossible de soumettre au laboratoire une éprouvette de sol aux chemins de contraintes ou de déformations réels induits sur site par un séisme. Des essais de laboratoire tels que triaxiaux de révolution, triaxiaux vrais, biaxiaux ou cylindre creux de torsion peuvent cependant permettre d'obtenir une bonne compréhension des mécanismes de base qui contrôlent le comportement cyclique des sols, et qui restent activés dans des sollicitations plus complexes que celles appliquées au laboratoire.

Le comportement mécanique des sables saturés sous sollicitations cycliques s'inscrit dans le cadre général mis en place pour l'étude du comportement sous chargement monotone, qui constitue le comportement $\alpha$ enveloppe $n$ du matériau, auquel il faut rajouter les spécificités liées au caractère cyclique des sollicitations appliquées. On va, en particulier, retrouver les notions de rupture de Mohr-Coulomb, d'état critique (ou état stable), d'état caractéristique et de surface d'effondrement ou surface d'instabilité, qui joueront un rôle essentiel dans les comportements observés sous chargement cyclique. Dans le cas cyclique comme dans le cas monotone, le comportement volumique du matériau (contractant ou dilatant) sera déterminant sur les réponses mécaniques observées, en particulier dans le cas non drainé. On présente tout d'abord quelques éléments relatifs au comportement drainé des sables, suivis d'une description des comportements non drainés (ou à volume constant) observables avec application aux phénomènes de mobilité cyclique et de liquéfaction cyclique " vraie »), responsables des dégâts occasionnés lors des séismes.

\section{1}

\section{Comportement cyclique drainé}

Sous sollicitation de cisaillement cyclique drainée, les sables vont généralement globalement se densifier lors de l'application des cycles successifs. Le mécanisme et l'ampleur de la densification sont contrôlés par les propriétés de contractance et de dilatance du matériau qui dépendent de son état initial, ainsi que, dans le cas cyclique, des caractéristiques de la sollicitation. L'état caractéristique (Luong, 1980) va jouer ici un rôle fondamental par sa propriété de seuil de délimitation intrinsèque entre un domaine contractant ( sub- caractéristique ») et un domaine dilatant ( $\propto$ sur-caractéristique »). Tant que le chemin des contraintes effectives correspondant à la sollicitation appliquée reste à l'intérieur du domaine sub-caractéristique, le matériau est contractant en charge (essentiellement élastique en décharge) et l'on assiste à une densification continue lors de l'application des cycles, avec durcissement progressif et stabilisation du matériau par adaptation ou accommodation. Dans le cas où le chemin des contraintes franchit en charge le seuil caractéristique, on observe alors une phase de dilatance qui est suivie, lors de la décharge suivante, par une phase de contractance accrue, accélérant le processus de densification. Le mécanisme de densification résultant d'une décharge à partir d'un état sur-caractéristique est induit par un important réarrangement des grains avec « réorientation » de la structure selon un mécanisme fortement irréversible (alors que l'élastoplasticité classique prévoierait une réponse élastique à la décharge). La figure 13 (Tatsuoka and Ishihara, 1974) permet de bien mettre en évidence, à partir d'essais réalisés à l'appareil triaxial, le mécanisme de densification, selon que les bornes des cycles en contrainte restent dans le domaine contractant (Fig. 13a) ou passent dans le domaine dilatant (Fig. 13b), avec une forte accélération du processus dans ce dernier cas (cas des sables denses). Pour un sable lâche, on aurait uniquement un mécanisme contractant (pas de dilatance). La figure 14 (Luong, 1980) présente une synthèse des comportements observés dans le cas du comportement drainé (ou sec) ainsi que dans le cas du comportement non drainé sur lequel on revient dans le paragraphe suivant. On remarque sur la figure que, pour ce qui est du comportement drainé, le seul cas pour lequel on n'a pas stabilisation du matériau est celui où la sollicitation appliquée serait entièrement contenue dans le domaine surcaractéristique, auquel cas on observerait le développement d'un mécanisme de rochet.

\section{9}

\section{Comportement cyclique non drainé : cas de la mobilité cyclique}

Dans le cas non drainé, les propriétés de contractance ou de dilatance du sable vont maintenant se traduire par une accumulation progressive de surpressions interstitielles d'origine " anélastique »), venant se rajouter à la surpression d'origine (élastique ». Cette accumulation des surpressions réduit d'autant le niveau des contraintes effectives régnant au sein du matériau, pouvant ainsi le conduire éventuellement jusqu'à la rupture. Deux mécanismes très différents, correspondant au cas des sables denses (dilatants en monotone) d'une part et au cas des sables lâches (uniquement contractants en monotone) d'autre part, vont pouvoir conduire à la ruine du matériau avec accumulation de grandes déformations. Dans les deux cas, le mécanisme de ruine (rupture) peut être directement relié au comportement observable sous chargement monotone. Dans le cas des sables denses, on parle de mobilité cyclique et, dans le cas des sables lâches, de liquéfaction cyclique ( vraie ». On s'intéresse tout d'abord, dans ce paragraphe, au cas de la mobilité cyclique. C'est ce phénomène, caractéristique des sables dans un état moyennement dense à très dense, qui a été, à l'origine, décrit et étudié par Seed dans ses travaux, puis par d'autres (en France, Mohkam. 1983) sous le nom de liquéfaction jusqu'à ce que ce phé- 

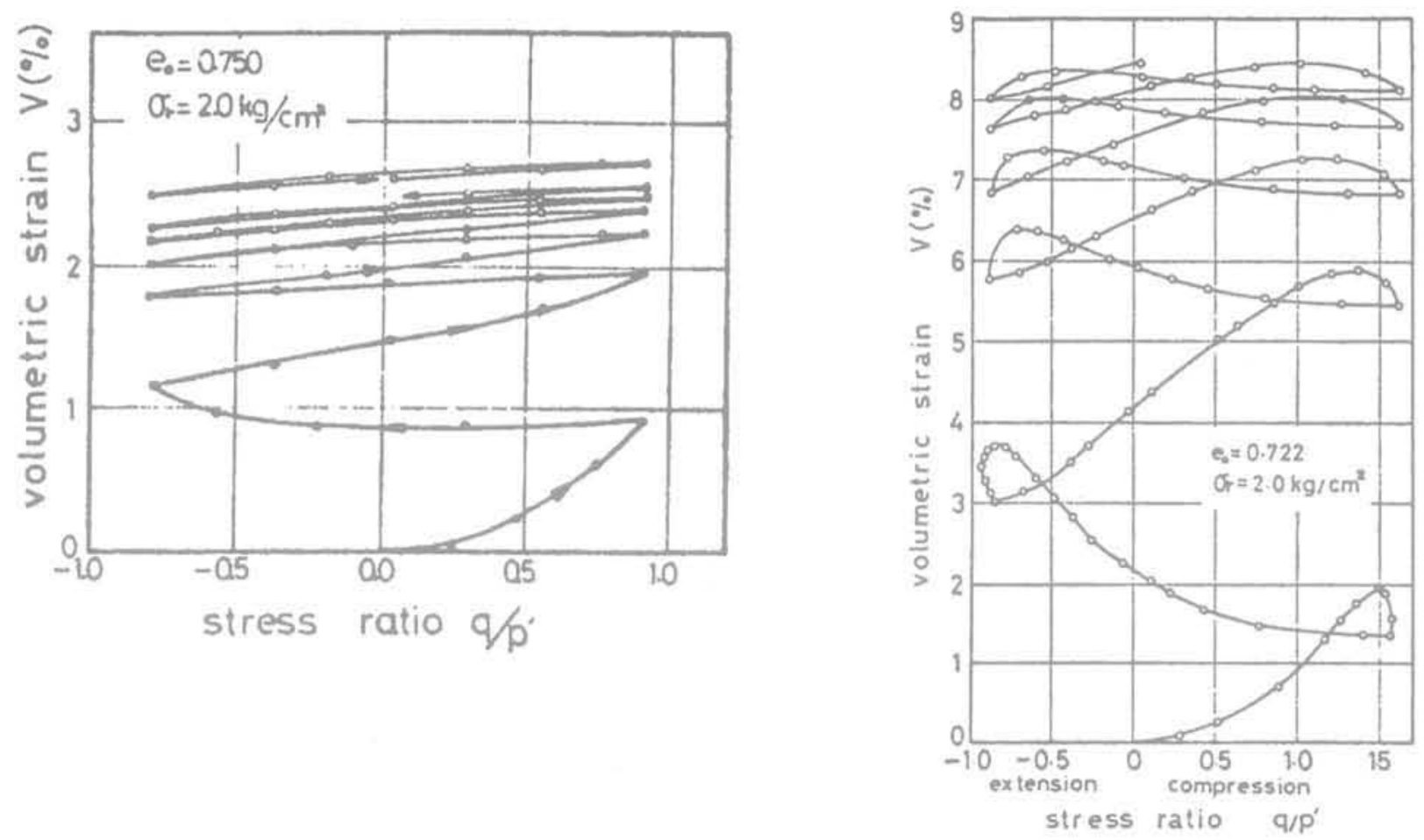

FG. 13 Mécanismes de densification d'un sable sous cisaillement cyclique à l'appareil triaxial (Tatsuoka et Ishihara, 1974).

Densification mechanisms of a sand submitted to a cyclic shear in the triaxial apparatus (Tatsuoka and Ishihara, 1974),

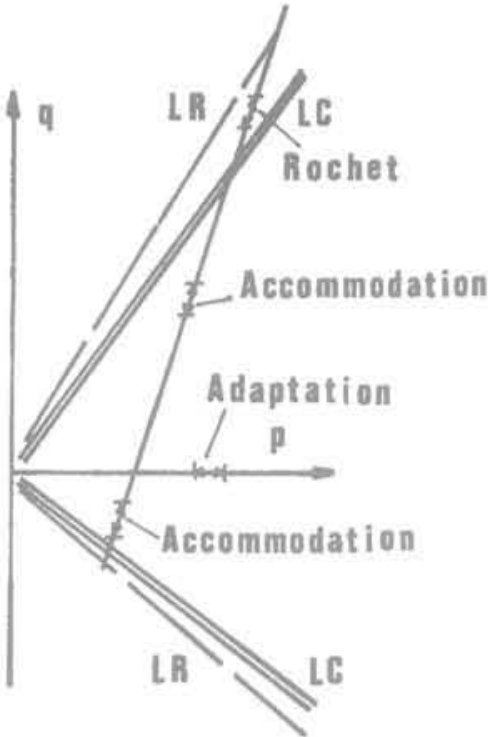

(a)

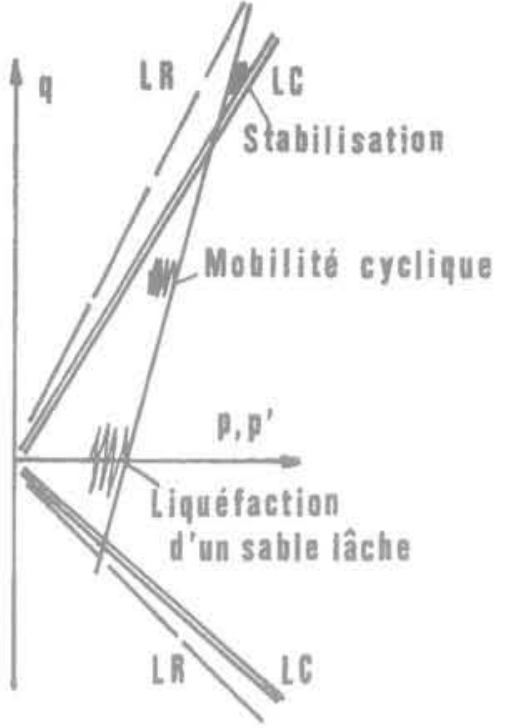

(b)

FiG.14 Cadre conceptuel proposé pour la prévision du comportement cyclique drainé (a) et non drainé (b) des sables (Luong, 1980).

Conceptual framework proposed for the prediction of the cyclic behaviour of sands in drained (a) and undrained (b) conditions (Luong, 1980).

nomène soit différencié du phénomène de liquéfaction vraie, au sens de l'instabilité, à partír des travaux de Castro (Castro, 1969 ; Castro et Poulos, 1977). Seed (1979) présente finalement une synthèse entérinant et distinguant clairement les deux phénomènes. La figure 15 montre un résultat typique de mobilité cyclique obtenu lors d'un cisaillement cyclique alterné non drainé réalisé à l'appareil triaxial sur le sable d'Hostun RF (Benahmed, 2001). Le comportement observé comporte deux régimes successifs bien distincts, avec une première phase, caractérisée par une génération progressive de surpressions interstitielles au sein de l'éprouvette dans un mécanisme " à un pic » par cycle, de très petites déformations sans pratiquement d'accumulation et donc stabilisation apparente du matériau sur une boucle d'accommodation très peu dissipative. Puis, dans une deuxième phase, on observe l'apparition rapide et l'établissement, en quelques cycles, d'un 

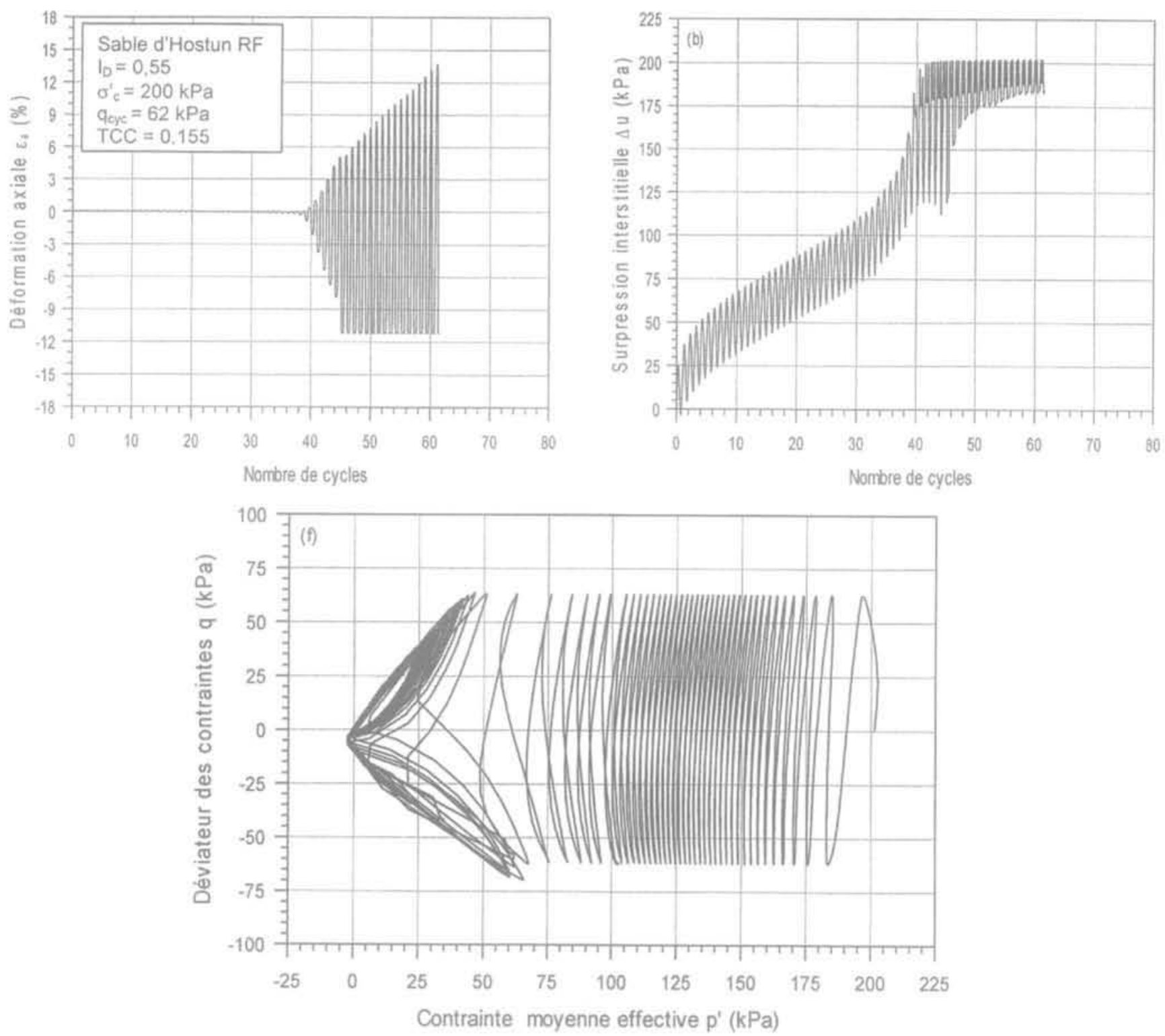

FG, 15 Comportement typique de mobilité cyclique tel qu'observé lors d'un essai triaxial alterné symétrique (Benahmed, 2001).

Typical cyclic mobility behaviour as observed in an alternated symetrical triaxial test (Benahmed, 2001).

deuxième régime, caractérisé par un mécanisme de génération de la surpression interstitielle « à deux pics ») avec accumulation rapide de déformations de grande amplitude lors de la succession des cycles suivants, caractéristique d'un phénomène de rochet. La représentation de l'essai dans le plan des contraintes (q, p') montre clairement que le deuxième régime est initié lorsque le chemin des contraintes effectives franchit pour la première fois le seuil caractéristique, généralement lors d'une phase d'extension à l'appareil triaxial, avec formation d'une première boucle de dilatance. A partir de là, et de manière analogue aux observations faites dans le cas drainé, les phases de décharge (en compression et extension) deviennent fortement contractantes (alors qu'elles étaient quasi élastiques dans le premier régime), entraînant le développement de fortes surpressions interstitielles d'origine plastique et une migration rapide du chemin des contraintes effectives vers l'origine et des valeurs très faibles de p'. En régime ultime « stabilisé $n$, on observe un mécanisme en $«$ ailes de papillon $»$, avec une boucle de dilatance ultime de compression et une boucle d'extension.
Ce mécanisme est caractérisé par deux passages, par cycle, par un état des contraintes effectives quasi nul ou nul, l'un en fin de décharge de compression et l'autre en fin de décharge d'extension (dans les deux cas, q et p' sont très proches de zéro). L'accumulation des grandes déformations se fait au passage par ces états ponctuels pour lesquels les efforts aux contacts intergranulaires sont très faibles. Lors de la recharge suivante, le matériau est ( restabilisé ») et regagne de la résistance en dilatance (durcissement), ce qui « pérennise » le mécanisme et permet de le reproduire un certain nombre de fois à l'appareil triaxial. L'essai, cependant, est arrêté lorsque les déformations axiales deviennent trop grandes et dépassent les capacités du dispositif. Le terme de «mobilité cyclique » provient de ce caractère répétitif observé à l'appareil triaxial avec accumulation séquentielle et « limitée » sur chaque cycle, de grandes déformations (mobilité).

L'initiation et le développement de la mobilité cyclique vont dépendre, en plus des paramètres intervenant de manière classique sur le comportement observé sous chargement monotone, de paramètres caractéri- 

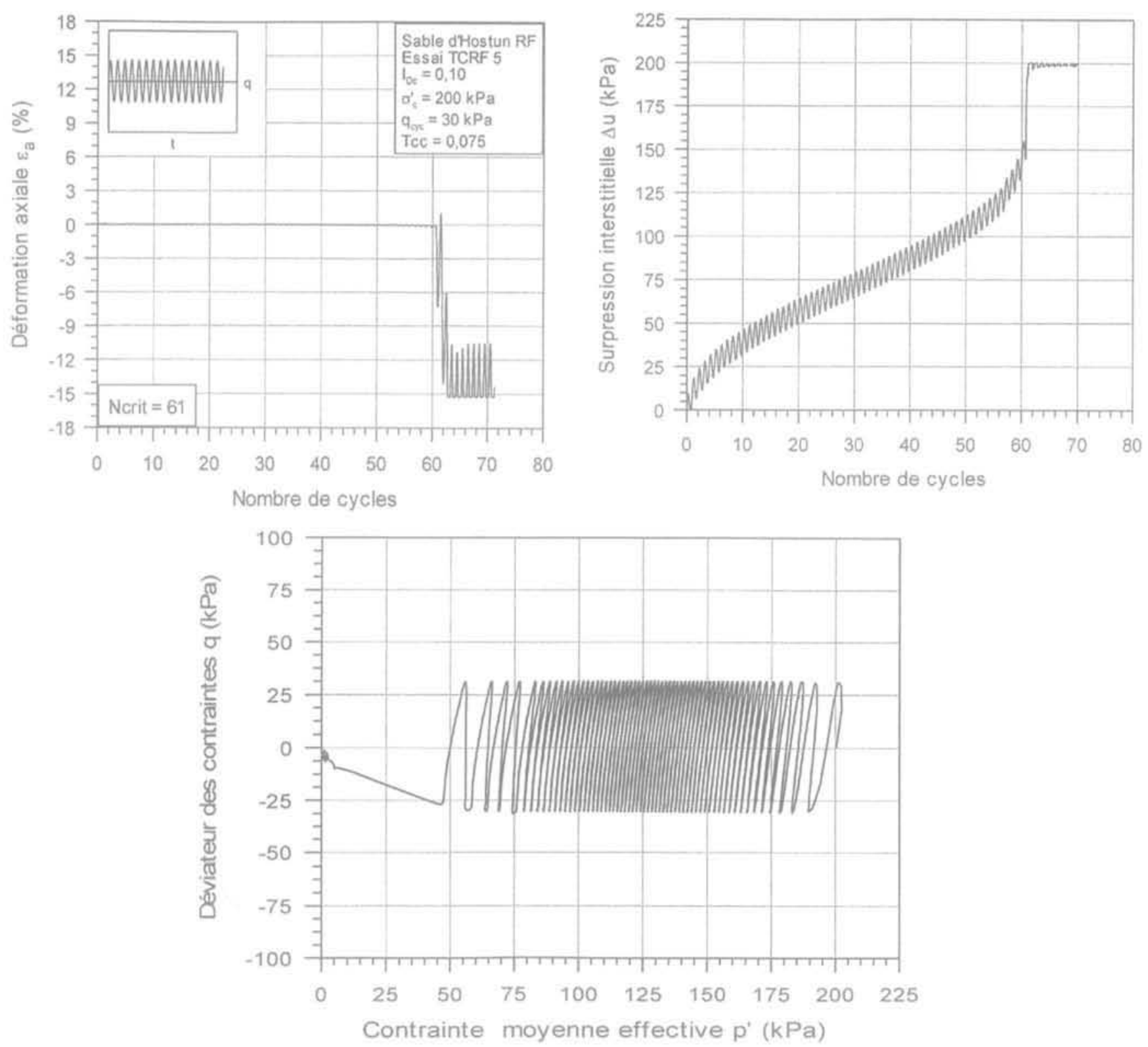

FiG.16 Comportement typique de liquéfaction cyclique vraie tel qu'observé lors d'un essai triaxial alterné symétrique (Benahmed, 2001).

Typical true cyclic liquefaction behaviour as observed in an alternated symetrical triaxial test (Benahmed, 2001).

sant la sollicitation cyclique (amplitude, caractère alterné ou non des cycles, symétrie, etc.) pour lesquels on donne quelques éléments dans le paragraphe 5.

\section{3}

\section{Comportement non drainé : cas de la liquéfaction cyclique " vraie "}

Dans le cas des sables dans un état lâche à très lâche, uniquement contractants, c'est un mécanisme différent de celui mis en jeu dans la mobilité cyclique, et très similaire à l'instabilité responsable du phénomène de liquéfaction " statique », qui va conduire, dans le cas cyclique, à la liquéfaction ( vraie » du matériau, au sens de l'écoulement. La figure 16 montre, dans les mêmes plans que ceux utilisés pour l'essai de mobilité cyclique, un résultat typique obtenu à l'appareil triaxial sur le sable d'Hostun pour un chargement alterné (Benahmed, 2001). On observe une première phase de l'essai au cours de laquelle la surpression interstitielle croît régulièrement de manière analogue à ce qui se passe pour le sable dense (avec un taux cependant supérieur) avec, en apparence, un comportement du type accommodé. Puis, pour un cycle particulier, appelé cycle critique, on observe, sur la courbe de cisaillement, une brusque chute de résistance avec développement rapide de très grandes déformations, accompagnée d'une forte augmentation de la surpression interstitielle au sein de l'éprouvette et apparition d'un état ultime à très faible résistance. On a alors interruption de l'essai par perte d'asservissement du dispositif. L'allure du chemin des contraintes effectives dans le plan $\left(q, p^{\prime}\right)$ montre que l'on a affaire, pour ce cycle critique, à un comportement très similaire au phénomène de liquéfaction statique décrit plus haut.

Ce type de comportement, appelé liquéfaction cyclique ("vraie ), correspond donc à un mécanisme d'instabilité, différent du mécanisme mis en jeu dans le phénomène de mobilité cyclique, et beaucoup plus dommageable que celui-ci. 
L'étude des seuils d'initiation du phénomène montre (Canou et al., 1994; Benahmed et al., 1999) que ceux-ci sont très similaires à ceux déterminés dans le cas monotone et que la notion de surface d'effondrement ou d'instabilité doit pouvoir s'appliquer ici aussi, avec les mêmes limitations que celles avancées pour le cas monotone. Il resterait à préciser si les seuils correspondant à la réponse cyclique sont exactement les mêmes que ceux correspondant au comportement monotone. En ce qui concerne les états ultimes obtenus après effondrement, on retrouve les notions d'état critique et d'état stable dont on a parlè, là aussi, pour le cas monotone.

Cornme pour la mobilité cyclique, l'amorce et le développement du phénomène de liquéfaction cyclique vont dépendre des mêmes paramètres que ceux intervenant dans le cas de la liquéfaction statique (comportement $\alpha$ enveloppe $»)$, combinés avec des paramètres liés aux caractéristiques de la sollicitation cyclique appliquée (amplitude, caractère alterné ou non, symétrie des cycles, nombre, etc.). On revient sur ce point dans le paragraphe suivant.

\section{5}

\section{Synthèse}

\section{des comportements observés. Recherche d'un cadre conceptuel global}

La description des comportements non drainés des sables reconstitués en laboratoire, sous cisaillement

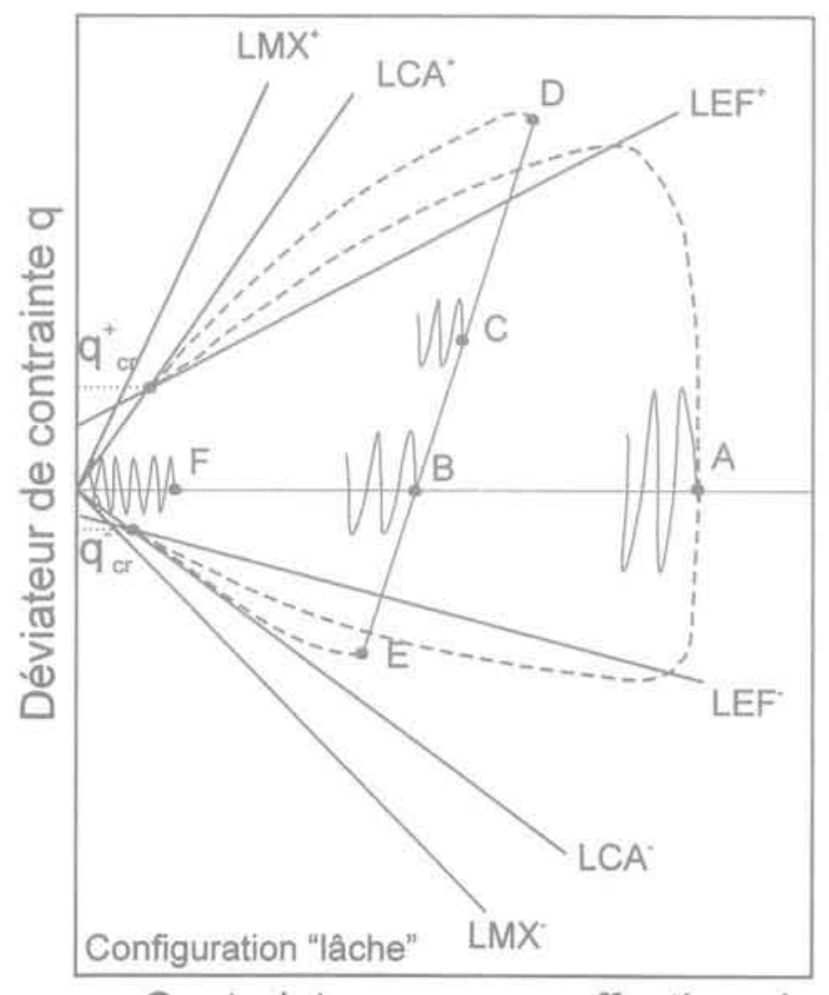

Contrainte moyenne effective $p^{\prime}$ monotone et cyclique, montre que ceux-ci sont assez divers, fortement non linéaires, mais obéissent à des règles assez précises contrôlées par leur comportement volumique. On constate, de plus, de fortes analogies entre comportement monotone et cyclique, le comportement monotone formant en quelque sorte une " enveloppe » pour le comportement cyclique. Ceci conduit à rechercher un cadre conceptuel global qui permettrait d'interpréter de manière cohérente, à partir de quelques concepts unificateurs, les comportements non drainés observés, à la fois sous chargement monotone et sous chargement cyclique. Dans le cas des sables de densité moyenne à forte, le diagramme de Luong (1980), basé sur le concept d'état caractéristique, (cas du triaxial, Fig. 15) fournit un cadre cohérent permettant de prévoir les comportements observables sous chargement monotone et cyclique non drainé (réponse cyclique du type mobilité cyclique). Il s'agit ici d'essayer de généraliser ce type de représentation pour pouvoir prendre en compte le cas des sables liquéfiables au sens de l'instabilité, dont la rupture sous cisaillement cyclique n'est plus contrôlée par l'état caractéristique mais par un seuil d'instabilité. La figure 17 montre un exemple possible d'une telle représentation, pour une configuration de sable dans un état lâche (Fig. 17a) et dans un état dense (Fig. 17b). L'idée est ici d'essayer d'englober à la fois les notions d'état caractéristique et de seuil d'effondrement en vue de prévoir l'initiation et le développement de la liquéfaction ou de la mobilité cyclique en fonction du point représentatif de l'état initial du matériau dans l'espace $\left(q, p^{\prime}, e\right)$, des caractéristiques de la sollicitation cyclique et des positions relatives du seuil caractéristique et du seuil d'instabilité. L'hypothèse retenue ici pour le seuil d'instabilité est celle de Sladen et al. (1985), qui définit

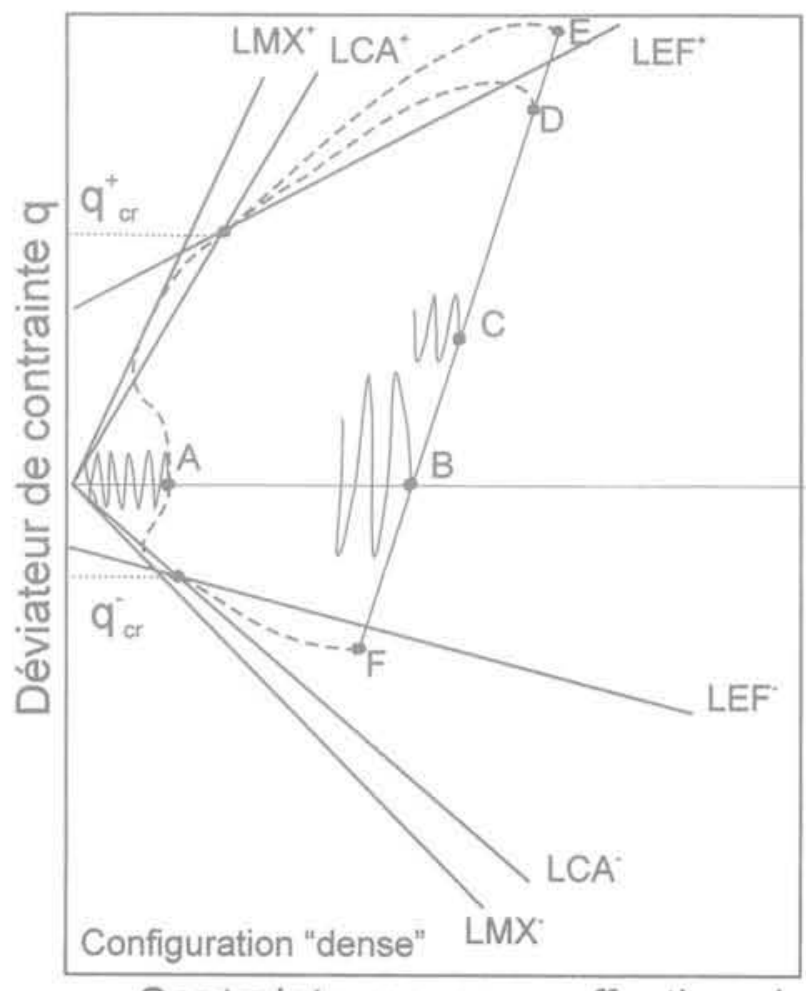

FG. 17. Cadre conceptuel global (schématique) de prévision du comportement non drainé d'un sable à l'appareil triaxial (configuration $\alpha$ lâche $n$ et $($ dense $n)$.

Global conceptual framework (schematic) for the prediction of the undrained behaviour of a sand, as observed in the triaxial apparatus ( $\alpha$ loose $»$ and $\alpha$ dense $»$ configurations). 
une droite d'effondrement ou d'instabilité (LEF) passant par le pic de résistance et l'état stable correspondant. Pour la configuration de type « lâche » (Fig. 17a), correspondant à de faibles ou très faibles niveaux de résistance mobilisables à l'état stable (éventuellement zéro), on aura généralement liquéfaction, sauf pour des états initiaux particuliers tels que le point $G$ sur la figure ou le point B (dans le cas de faibles amplitudes de sollicitation). Pour la configuration du type $\approx$ dense », on aura généralement mobilité cyclique (et I’on retrouve dans ce cas la configuration de Luong, Fig. 14) sauf pour des états initiaux tels que les points D, E ou $\mathrm{G}$, qui correspondront généralement à de forts niveaux de consolidation (isotrope ou anisotrope), pour lesquels le chemin de contrainte rencontrera d'abord la droite d'instabilité (LEF) et non le seuil caractéristique (LCA). Il est clair que si l'on prend une autre hypothèse sur le seuil d'instabilité (par exemple, passage du seuil par l'origine des axes), un autre schéma devra être imaginé pour rendre compte des différents comportements observables. Il est à noter, par ailleurs, que, si le seuil caractéristique peut être, avec une assez bonne approximation, considéré comme unique (l'angle caractéristique peut être assimilé à l'angle de frottement critique), il est nécessaire de donner une description de l'évolution de l'inclinaison de la droite d'instabilité pour des états initiaux situés en limite d'une plage moyenne et l'on n'a donc pas unicité du seuil d'instabilité (Canou et al., 1990).

\section{6}

\section{Conclusion}

On a tenté de faire le point, dans ce texte, sur les mécanismes et comportements mécaniques élémentaires qui sont à l'origine des ruptures et des dégâts souvent spectaculaires constatés, généralement sur des sites saturés à dominante sableuse (naturels ou artificiels tels que barrages en terre, îles artificielles), lors de l'application de sollicitations rapides telles que séismes, raz-de-marée, chocs importants, etc. Ces ruptures, connues sous le nom générique de (liquéfaction $»$, ont pour origine une perte importante et soudaine de la résistance au cisaillement du sol résultant d'une génération de surpressions interstitielles élevées entraînant une chute importante du niveau des contraintes effectives régnant au sein du matériau, qui va entraîner un comportement temporairement semblable à celui d'un liquide épais, avec toutes les conséquences associées (écoulements et déplacements importants du sol, lorsque les configurations s'y prêtent, avec entraînement éventuel d'ouvrages, perte de portance pour les structures de génie civil, avec enfoncement et basculement, etc.). Dans le cas cyclique, deux phénomènes bien distincts, à savoir la liquéfaction a vraie $»$ et la mobilité cyclique, peuvent être à l'origine de cette perte de résistance, Dans les deux cas, les mécanismes élémentaires correspondants sont caractérisés par un comportement à volume constant ou pratiquement constant (faible compressibilité), qui est généralement observé en mécanique des sols pour les matériaux saturés en comportement non drainé.

La liquéfaction ( vraie ») est de loin le phénomène le plus dangereux, et correspond à une instabilité parti- culière caractéristique des sables uniquement contractants (en général, structures lâches à très lâches), susceptibles de développer des surpressions interstitielles très élevées en non drainé. Il peut être initié sous un chargement monotone ou cyclique (quasi statique ou dynamique) et va, lorsque la configuration s'y prête, se développer sous forme d'un écoulement, avec chute rapide et pérenne de résistance au cisaillement non drainé jusqu'à une valeur ultime de très faible niveau (voire nulle dans le cas de la liquéfaction totale). C'est ce phénomène qui sera par exemple à l'origine des écoulements quasi spontanés qui sont parfois observés dans des massifs naturels ou artificiels et qui correspondent à un état initial très instable du matériau.

La mobilité cyclique, quant à elle, est caractéristique des structures dilatantes (sables moyennement denses à très denses) et ne peut se développer que sous chargement cyclique (quasi statique ou dynamique), avec un mécanisme de génération de fortes surpressions interstitielles de contractance lors des phases de décharge au cours de l'application de la sollicitation. Ce type de comportement, qui ne constitue pas une instabilité au sens utilisé ci-dessus, se traduit par l'apparition d'états transitoires du matériau à contrainte effective nulle ou quasi nulle pouvant quand même entrainer, si la configuration s'y prête, des dégâts importants (enfoncement et basculement d'ouvrages). Un écoulement au sens de la liquéfaction "vraie " décrite ci-dessus ne pourra cependant pas se développer dans un tel matériau (blocage de l'écoulement par dilatance).

En situation réelle, le déclenchement et le développement d'écoulements dans un massif sableux est un problème très complexe à prévoir, dans la mesure où, bien que contrôlés à la base par les mécanismes décrits ci-dessus, les phénomènes d'écoulement vont aussi fortement dépendre des conditions spécifiques existant sur le site, en termes d'état initial, d'hétérogénéité, de conditions aux limites, autant de facteurs souvent difficiles à évaluer précisément. Le caractère souvent régressif des écoulements ainsi que les phénomènes de redistribution et de dissipation des surpressions interstitielles au sein du matériau viennent aussi fortement compliquer l'analyse d'un problème réel.

On n'a pas abordé, dans ce texte, le problème de la modélisation des comportements observés, qui constitue en soi un vaste domaine, l'objectif final étant la prévision du comportement des massifs dans des problèmes aux limites réels. Il convient simplement de noter qu'il existe actuellement un assez grand nombre de modèles $\alpha$ macroscopiques $)$, que l'on peut regrouper en différentes familles, qui permettent de rendre compte des comportements décrits ci-dessus, sous chargement monotone et cyclique, tout d'abord sur essai homogène puis dans des problèmes aux limites plus complexes, mais que l'exercice dans ce dernier cas reste très difficile. Les modèles basés sur des approches du type micro-macro commencent à se développer dans ce domaine et constituent une voie certainement prometteuse en vue de mieux comprendre les phénomènes physiques qui sont à l'origine des comportements macroscopiques observés, ainsi qu'un terrain de collaboration privilégié entre mécaniciens des sols, mécaniciens et physiciens des milieux granulaires. 
Benahmed N. - a Comportement mécanique d'un sable sous cisaillement monotone et cyclique : application aux phénomènes de liquéfaction et de mobilité cyclique $¥$. These de doctorat de I'ENPC, 2001, 351 p.

Benahmed N., Canou J. Dupla J.-C. $\alpha$ Influence des conditions initiales et du type de chargement sur la stabilité du comportement non drainé d'un sable lâche n. C.R. 12th Europ. Conf. of Soil Mechanics and Found, Eng. Amsterdam, vol. 2, 1999, p. 687-690.

Bjerrum L., Krinstad S., Kummeneje O. $\alpha$ The shear strength of a fine sand $x$. Proc. 5th Intern. Conference on Soil Mechanics and Found. Eng., Paris, vol. 1, 1961, p. 29-37

Blondeau F. - a Incidence de l'anisotropie de consolidation sur le potentiel de liquéfaction statique ; application au glissement sous-marin du port de Dunkerque ». Revue francaise de géotechnique, $n^{\circ} 37,1986, \mathrm{p} .72-80$

Canou J. - k Liquéfaction statique des sables effondrables $\mathrm{n}$. Conférence au Comité français de mécanique des sols, octobre, Paris, 1987.

Canou J, - $\alpha$ Contribution à l'étude et à l'évaluation des propriétés de liquéfaction d'un sable x. Mémoire de thèse de doctorat de I'ENPC, 1989, $337 \mathrm{p}$.

Canou J. El Hachem M. Kattan A. - « Propriétés de liquéfaction statique d'un sable làche m, Les Cahiers de Phéologie, 8, n 4, 1990, p. 207-218.

Canou J., Bahda F., Saitta A., Dupla J.-C. « Initiation de la liquéfaction des sables sous chargement monotone et cycliquex. Proc. 13th Intern. Conference on Soil Mechanics and Found. Eng.. New Delhi, 1994, p. 1297-1300.

Canou J., Thorel L., de Laure E.-unfluence d'un déviateur cie contrainte initial sur les caractéristiques de liquéfaction statique d'un sable w. Proc. 10th Europ. Conf. On Soil Mechanics and Found. Eng., vol. 1, 1991, p. 49-52

Casagrande A. - $x$ Liquefaction and cyclic deformation of sands, a critical review n: 5th Pan American Conf. on Soil Mechanics and Found. Eng., Buenos Aires, vol. 5, 1975, p. 79-133

Castrò G. - u Liquefaction of sands n. Ph.D. thesis, Harvard Soil Mechanics Series, $n^{\circ} 81$, Harvard Univ., Cambridge, USA, $1969,112 \mathrm{p}$

Castro G., Poulos S.J. - « Factors affecting liquefaction and cyclic mobility 1, Journ. of the Geotechnical Eng. Div., ASCE, 103, n*6, 1977, p. 501-515

Darve F. - « Liquefaction phenomenon : modelling, stability and uniqueness x. Proc Intern Conf on Numerical Analysis of Liquefaction Problems, Davis, USA, 1993. p. 1305-1319.

Darve F., Laouafa F. - $\alpha$ Liquéfaction et stabilité dynamique $n$. CR $5^{*}$ Colloque National de I'AFPS, Cachan, 1999, p.123132.

Drucker D.C. - a A more fundamental approach to stress-strain relations $x$. Proc. First US Nation. Congress of Applied Mechanics, 1951, p. 487-491.
Dupla J.-C., Canou J., Normand P., Piffer L_ a Influence of loading path on the lique. faction characteristics of a sand $n$. 11th Europ. Conf. on Soil Mechanics and Found. Eng. Copenhacue, vol. 3, 1995, p. 55-60.

Flavigny E. Foray P. - « Comportemen mécanique des sables sur chemins non drainés et liquéfaction $n$. "1" colloque national de génie parasismique, StRémy-lès-Chevreuse, 1986

De Gennaro V., Canou J., Dupla J.-C. * Conditions of initiation of liquefaction of a loose sand under cvelic loading $p$ Proc. 7 th Australia-New Zealand Conf. on Geomechanics, Adelaide, 1996. p. 299-303.

Habib P. - "Liquéfaction des sables $*$. Annales de ITTBTP, $n^{e} 355,1977$, p. 155. 157

Hazen - a Hydraulic fill dams ». ASCE transactions, 83, 1920, p. 1713-1745,

Highter W.H. Tobin R.F. - u Flow slides and the undrained brittleness index of some mine tailings n. Mechanics of land. slides and slope stability, S,L. Koh Eng. Geologv edit., 1980, p. 71-82.

Hill R. - a A general theory of uniqueness and stability in elasto-plastic solids $p$ Journal of Mechanics and Physics of Solids, 6, 1958, p. 236-249

Ibraim E., Doanh T. - « Minimum undralned strength of anisotropically consolidated loose Hostun sand RF p. Proc. Conf. on Deformation and Progressive Failure in Geomechanics, Nagoya, 1997. p. $623-628$

Ishihara K. - $\alpha$ Liquefaction and flow failure during earthquakes y. Géotechnique, 43, n³, 1993, p. 351-415 (The Rankine Lecture)

Konrad J.-M. - " Minimum undrained strength versus steady-state strength of sands .). Journ of Geotechn. Eng Div. ASCE, vol. 116, n०6, 1990, p. 948-963. Konrad J.-M., Flavigny E., Mégachou M. ๔ Comportement non drainé du sable d'Hostun lấche x. Revue française de géotechnique, $\mathrm{n}^{\circ} 54,1991$, p. 53-64.

Koppeian A.W Van Wamelen B.M. Weinberg L.J.H. - a Coastal landslides in the Dutch province of Zealand 1 . Proc. 2nd Intern. Conf. on Soil Mech. and Found. Eng., Rotterdam, 1948, p. 89-96.

Kramer S1 Seed H B - - Initiation of soi liquefaction under static loading conditions v. Journ. of Geotechnical Eng. ASCE, vol. 114, n 4, 1988, p. 412-430.

Lade P.V. - " Static instability and liquefaction of loose fine sandy slopes ?. Journa! of Geotechnical Eng., ASCE, vol. 118, $n^{\circ} 1,1992$, p. 51-71.

Ladie P. V Nelson R.B. Marvin Ito Y $\alpha$ Instability of granular materials with non associated flow n. Journal of Eng. Mechanics, vol. 114, n $12,1988, p, 2173-$ 2191.

Lade P.V.. Pradel D. - $\pi$ Instability and plas tic flow of soils. I: experimental observations 1. Journal of Eng. Mechanics. vol. 116, n० 11, 1990, p. 2532-2550.

Lanier J., Di Prisco C. Nova R. - "Étude expérimentale et analvse théorique de l’anisotropie induite du sable d'Hostun $n$. Revue française de géotechnique, $n^{\circ} 57,1991$, p. $59-74$
Luong M.P. - " Phénomènes cycliques dans les sols pulvérulents $x$. Revue francaise de géotechnique, ${ }^{\circ} 10,1980$, p. 39 . 53.

Matiotti R. Ibraim E., Doanh T. - « Com portement non drainé du sable d'Hostun RF très láche en condition anisotrope n. Revue francaise de géotechnique, $\mathrm{n}^{\circ} 75,1996$, p. 35-46.

Méqachou M. - a Stabilité des sables lâches : essais et modélisations 10 . Thèse de doctorat, Université de Grenoble. 1993.

Mohkam M. - « Contribution à l'étude expérimentale et théorique du comportement des sables sous chargements cycliques ». Thèse die doctorat de I'Université de Grenoble, 1983, 232 p.

Poulos S.J. - " The steady state of deforma. tion w. Journ. of the Geotechn. Eng. Div. ASCE, vol. 107, n० GT5, 1981, p. 553-562.

Roscoe K.H. Schofield A.N., Wroth C.P. "On the yielding of soils s. Geotechnique, vol. $8, n^{\circ} 1.1958$, p. 22-52.

Schlosser F. - x Liquéfaction de veines de sable làche dans des talus sous-marins D. C.R. 11th Intern. Conference on Soil Mechanics and Foundation Engin., San Francisco, 1985

Schofield A.N., Wroth C.P. $-\alpha$ Critical state soil mechanics x. MCGraw-Hill, London, 1968.

Seed H.B. - $"$ Landslides during earthguakes 1 . Journ of the Soil Mechanics and Foundation Div., ASCE, vol. 94, n5. 1968, p. 1053-1122

Seed H.B. - a Soil liquefaction and cyclic mobility evaluation for level ground during earthquakes n. fourn of the Geotechnical Eng. Div., ASCE, vol. 105, n² 1979, p. 201-255

Seed H.B. - a Stability of port fills and coastal deposits $\%$. 7 th Asian Regiona. Conf on Soil Mechanics and Found. Eng., vol. 2, 1983, p. 31-41.

Seed H.B., Idriss I.M. - $\propto$ Analysis of soil liquefaction: Niigata earthquake x. Journ, of the Soil Mechanics and Foundation Div., ASCE, vol. 93, n 3,1967, p. 83-10B.

Sladen J.A., d'Hollander R.D., Krahn J. (The liquefaction of sands, a collapse surface approach p. Canadian Geotechnical Journal, vol. 22, 1985, p. 564-578.

Tatsuoka F. Ishihara K. - « Drained deformation of sand under cyclic stresses reversing direction $x$. Soils and Foundations, vol. 14, n'3, 1974, p. 51-65.

Terzaghi K. - « Erdbaumechanik auf Badenphysikalischer Grundlage 1 . Deuticke Edit., Vienna, 1925.

Terzaghi K. - "Varieties of submarine slope failures 1). Harvard Soil Mech. Series, $n^{\circ}$ 52, 1956, $16 \mathrm{p}$

Vaid Y.P., Chern J.C. - " Cyclic and monotonic undrained response of saturated sands 1 . Advances in the art of testing soils under cyclic loading, 1985, p. 120 147

Verdugo R.L. - « Characterization of sandy soilbehaviour under large deformation . Ph.D. dissertation, University of Tokyo, 1992, 455 p. 